\title{
Exploring the Mechanism of Edaravone for Oxidative Stress in Rats with Cerebral Infarction Based on Quantitative Proteomics Technology
}

\author{
Guozuo Wang, ${ }^{1}$ Xiaomei Zeng, ${ }^{2}$ Shengqiang Gong, ${ }^{1}$ Shanshan Wang, ${ }^{1}$ Anqi Ge, ${ }^{3}$ \\ Wenlong Liu, ${ }^{1}$ Jinwen Ge $\mathbb{D D}^{1}{ }^{1}$ and Qi $\mathrm{He}^{2}$ \\ ${ }^{1}$ Hunan University of Chinese Medicine, Changsha, Hunan, China \\ ${ }^{2}$ People's Hospital of Ningxiang City, Ningxiang, Hunan, China \\ ${ }^{3}$ The First Affiliated Hospital of Hunan University of Chinese Medicine, Changsha, Hunan, China
}

Correspondence should be addressed to Jinwen Ge; 001267@hnucm.edu.cn

Received 3 August 2021; Revised 23 October 2021; Accepted 10 November 2021; Published 4 January 2022

Academic Editor: Thanasekaran Jayakumar

Copyright ( $\odot 2022$ Guozuo Wang et al. This is an open access article distributed under the Creative Commons Attribution License, which permits unrestricted use, distribution, and reproduction in any medium, provided the original work is properly cited.

\begin{abstract}
Objective. To explore the mechanism of edaravone in the treatment of oxidative stress in rats with cerebral infarction based on quantitative proteomics technology. Method. The modified Zea Longa intracavitary suture blocking method was utilized to make rat CI model. After modeling, the rat was intragastrically given edaravone for 7 days, once a day. After the 7 -day intervention, the total proteins of serum were extracted. After proteomics analysis, the differentially expressed proteins are analyzed by bioinformatics. Then chemoinformatics methods were used to explore the biomolecular network of edaravone intervention in CI. Result. The neurological scores and pathological changes of rats were improved after the intervention of edaravone. Proteomics analysis showed that in the model/sham operation group, 90 proteins in comparison group were upregulated, and 26 proteins were downregulated. In the edaravone/model group, 21 proteins were upregulated, and 41 proteins were downregulated. Bioinformatics analysis and chemoinformatics analysis also show that edaravone is related to platelet activation and aggregation, oxidative stress, intercellular adhesion, glycolysis and gluconeogenesis, iron metabolism, hypoxia, inflammatory chemokines, their mediated signal transduction, and so on. Conclusion. The therapeutic mechanism of edaravone in the treatment of CI may involve platelet activation and aggregation, oxidative stress, intercellular adhesion, glycolysis and gluconeogenesis, iron metabolism, hypoxia, and so on. This study revealed the serum protein profile of edaravone in the treatment of cerebral infarction rats through serum TMT proteomics and discovered the relevant mechanism of edaravone regulating iron metabolism in cerebral infarction, which provides new ideas for the study of edaravone intervention in cerebral infarction and also provides reference information for future research on the mechanism of edaravone intervention in iron metabolism-related diseases.
\end{abstract}

\section{Introduction}

Stroke is the second most common cause of death in the world, and it is also the main cause of adult disability [1]. As the American Heart Association and the National Institute of Neurological Diseases and Stroke reported, about 795,000 Americans suffer from ischemic stroke (such as cerebral infarction, CI) each year, and 220,000 die from ischemic stroke each year $[2,3]$. The "China Cardiovascular Disease Report 2018" compiled by the National Center for Cardiovascular Diseases showed that the prevalence of cardiovascular disease in China is on the rise. It is estimated that there are 290 million people suffering from cardiovascular disease, of which 13 million have stroke and 11 million have coronary heart disease [4]. According to the China Stroke Prevention Report (2015), about $15 \%$ of people over 40 years old are high-risk groups. From 2011 to 2013, the comprehensive standardized prevalence rate of ischemic stroke was about $2 \%$, and the prevalence rate increased by $8.1 \%$ [5]. According to data from the World Bank, by 2030, China will have 31.77 million patients with ischemic stroke, which will cost US $\$ 40$ billion each year [6]. Current research 
showed that oxidative stress plays an important role in the pathological process of ischemic brain injury. After cerebral ischemia, the production of superoxide anions, hydrogen peroxide, hydroxyl free radicals and peroxynitrite anions increases due to factors such as excitotoxicity, inflammatory response, and hypoxic environment inhibiting cell respiration, especially during reperfusion [7-9]. Hydroxyl free radicals and peroxynitrite anions can promote protein nitrification and oxidation, lipid peroxidation, mitochondrial and DNA damage, inflammatory activation, cell necrosis, and apoptosis, which can cause brain damage $[10,11]$.

As a scavenger of oxidative stress, edaravone is widely used in China and other Asian countries, but it has not been approved for use in Western countries [12]. A systematic review involving 49 animal experiments showed that the functional and cognitive prognosis of edaravone in an animal model of focal cerebral ischemia increased by $30.3 \%$ and $25.5 \%$, respectively [13]. A systematic review published by Yang et al. included 18 randomized controlled trials involving 1802 patients, and the results showed that edaravone can significantly reduce the mortality or long-term disability rate of acute ischemic stroke (AIS) [14]. Both of these systematic reviews support the effectiveness of edaravone for acute ischemic stroke. Although the current research shows that edaravone can mainly improve the oxidative stress in CI [15], there is no systematic research on the mechanism of edaravone in the treatment of ischemic brain injury such as proteomics and chemoinformatics. In particular, with the rapid development and rapid iteration of current proteomics technology, the large amount of data obtained from proteomics detection and analysis represents all the processes and changes that occur in the cell $[16,17]$. Therefore, in this research, proteomics and bioinformatics analysis strategies would be utilized to observe the changes of related indicators after edaravone in the treatment of CI and further explore the mechanism of edaravone to protect ischemic brain injury.

\section{Material and Methods}

2.1. Experimental Materials. SPF-grade male SD rats, weighing 200 230 g, were provided by Laboratory Animal Technology Co., Ltd., and the certificate number is SCXK: (Xiang) 2017-0012 (edaravone injection, article number, company batch number $1511001 \mathrm{Y}$ ). RIPA lysate was obtained from Thermo Inc.; iodoacetamide was obtained from IAM, Sigma Aldrich; trypsin was obtained from Promega; ammonium bicarbonate was obtained from Sigma Aldrich Inc.; SOD, MDA, and GSH-px kits were obtained from Shanghai Enzyme Linked Biotechnology Co., Ltd.; rat angiotensinogen (AGT) ELISA kit (E-EL-M0013c) was purchased from Elabscience Inc. The Catalase (CAT) kit was purchased from Nanjing Jiancheng Biotechnology Research Institute. Protein quantification kit, Exactive Plus mass spectrometer, EASY-n LC 1000 liquid analyzer, C18 analytical column, EASY-Spray Column, AcclaimPep Map 100 were obtained from Thermo Inc. Nano Vue UV-Visible Spectrophotometer was obtained from GE Healthcare. Laser Doppler flow meter was obtained from Mo or VMS-LDF, Moor Instruments, Aminster, Devon, UK.

\subsection{Experimental Methods}

2.2.1. Animal Grouping and Intervention. Thirty male healthy SD rats were randomly divided into 3 groups by random number table method: sham operation group (CN group), CI model group (CI group), and edaravone group (CE group), 10 rats in each group. The rats in the model administration group were given edaravone by gavage with a dose of $3.5 \mathrm{~g} / \mathrm{kg}$. The sham operation group and the model group were given distilled water by gavage with a volume of $10 \mathrm{~mL} / \mathrm{kg}$. The drug was administered once a day at $14: 00$ for 7 consecutive days. All animals' care and experimental procedures were approved by the Animal Ethics Committee of Hunan University of Chinese Medicine and were in accordance with the National Institute of Health's Guide for the Care and Use of Laboratory Animals.

2.2.2. Animal Modeling. After drug administration, according to the method of Longa et al. [18], the right middle cerebral artery occlusion (MCAO) model of rats was prepared by the suture method. First, the rats were anesthetized by intraperitoneal injection of $1 \%$ sodium pentobarbital $(50 \mathrm{mg} / \mathrm{kg})$, and then the right common carotid artery was carefully separated. Then, a 5-0 suture was used to insert a blunt nylon thread from the right external carotid artery of the rat. The thread enters the intracranial segment of the internal carotid artery from the side of the external carotid artery through the bifurcation of the common carotid artery and the extracranial segment of the internal carotid artery and reaches the branch of the middle cerebral artery for circular ligation. During the model preparation process of rats in the sham operation group, except that the nylon thread did not block the middle cerebral artery, the other operations were the same as those in the model group and edaravone group. After the rats were awake, the neurological function was scored according to Longa's 5-point system.

Zero points meant no neurological deficits. One point meant mild loss of nerve function: limited extension of the left forelimb, flexion when the tail is lifted. Two points meant moderate neurological deficit: rotating to the paralyzed side (left side) when crawling. Three points meant severe neurological deficit: falling into the paralyzed side (left side) while crawling. Four points meant difficulty in crawling and performance of decreased consciousness. Rats with a score of 1 to 3 were included in the experiment.

2.2.3. Immunofluorescence Staining. After BrdU was dissolved in normal saline, the dose was determined at $100 \mathrm{mg} /$ $\mathrm{kg} / \mathrm{d}$, and intraperitoneal injection was performed. The sections of brain tissue were immersed in 3\% $\mathrm{H} 2 \mathrm{O} 2$ deionized water for $10 \mathrm{~min}$, washed with PBS for $5 \mathrm{~min} \times 3$ times, and immersed in $2 \mathrm{~mol} / \mathrm{L} \mathrm{HCl}$ at $37^{\circ} \mathrm{C}$ for $15 \mathrm{~min}$. Then, the sections were washed with PBS for $5 \mathrm{~min} \times 3$ times; $5 \%$ goat serum was blocked at room temperature for $30 \mathrm{~min}$, 
and the liquid was aspirated. After that, BrdU monoclonal antibody $(1: 100)$ of $10 \mu \mathrm{L}$ was added and incubated in $37^{\circ} \mathrm{C}$ water bath. Rhodamine (light emission wavelength $570 \sim$ $590 \mathrm{~nm}$, red light) staining was performed, $37^{\circ} \mathrm{C}$ water bath for $30 \mathrm{~min}$. Finally, the slices were packaged with glycerin and observed with an OLYMPUS BX51 fluorescence microscope by the corresponding color filters at 520 and $580 \mathrm{~nm}$, respectively. Rhodamine is red.

\subsection{Protein Sample Processing}

2.3.1. Serum Protein and Brain Tissue Protein Extraction. After the rats were anesthetized with $1 \%$ pentobarbital sodium, blood was taken from the abdominal aorta, and the preserum was left standing. The rats were then sacrificed by cervical dislocation under anesthesia. Then, the brain tissue was taken out and washed with physiological saline, the brain tissue was put in the EP tube, RIPA lysis solution was added, and the brain tissue was cut into pieces with ophthalmological scissors and put it in a glass homogenizer for homogenization. The brain tissue homogenate was placed in a centrifuge at $4^{\circ} \mathrm{C}$ and centrifuged at $13,000 \mathrm{r} / \mathrm{min}$ for 30 minutes, and the precipitate was discarded. The upper liquid was the whole brain tissue protein.

2.3.2. Enzymatic Hydrolysis of Protein. The protein sample was added with TCEP with a final concentration of $5 \mathrm{mmol} / \mathrm{L}$, $37^{\circ} \mathrm{C}$ water bath for 30 minutes, and allowed to cool in room temperature. IAA with a final concentration of $10 \mathrm{mmol} / \mathrm{L}$ was added and kept in a $37^{\circ} \mathrm{C}$ dark water bath for 30 minutes. The enzyme solution was added to a water bath at a ratio of protein content to trypsin solution of $25: 1$ at $37^{\circ} \mathrm{C}$ overnight. On the second day, formic acid was added to the digested protein sample, and $0.1 \%$ was added to terminate the digestion reaction. The digested product was transferred to a 10 $\mathrm{kDa}$ ultrafiltration tube and centrifuged at $4^{\circ} \mathrm{C}$ at $10,000 \mathrm{r} /$ $\mathrm{min}$ for $30 \mathrm{~min}$. The protein in the lower layer of the ultrafiltration tube can be directly analyzed by mass spectrometry.

2.3.3. TMT Labeling and High-Performance Liquid Chromatography (HPLC) Classification. The protein extract was used to remove the high-abundance proteins, and the BCA kit was used to determine the protein concentration. $20 \mu \mathrm{g}$ of the eluate was taken for SDS-PAGE electrophoresis to detect the removal of high-abundance proteins. Trypsin enzymatically hydrolyze peptides with Strata X C18 (Phenomenex) desalting and freeze-drying in vacuum. The peptide was dissolved with $0.5 \mathrm{M}$ TEAB, and the peptide was labeled according to the TMT kit operating instructions. The peptides were fractionated by high $\mathrm{pH}$ reverse HPLC, and the column was Agilent 300Extend C18.

2.3.4. LC-LTQ-MS/MS Analysis and Mass Spectrum Data Retrieval. In mass spectrometry analysis, the peptides were separated using the EASY-nLC 1000 ultra-high performance liquid system, and the Thermo ScientificTM Q ExactiveTM Plus was simultaneously used for detection and analysis. The peptides are separated and ionized and then enter the $Q$ ExactiveTM Plus mass spectrometer for analysis. Peptide precursor ions and their secondary fragments are detected and analyzed by Orbitrap. The secondary mass spectrum data is retrieved by Maxquant (v1.5.2.8), and the mass spectrum quality control is performed at the same time.

2.4. Bioinformatics and Chemical Informatics Analysis. All the proteins retrieved from the database are analyzed, and the proteins whose expression changes are more than 1.3 times (fold change greater than 1.5 for upregulation and less than 0.67 for downregulation) are selected as differential proteins. Pubchem (https://pubchem.ncbi.nlm.nih.gov/) was used to retrieve the molecular structure of edaravone and saved as the "sdf" structure. Then, it was imported into Pharmmapper (http://lilab-ecust.cn/pharmmapper/) for potential target prediction [19]. The GeneCards database (https://www.genecards.org/) [20] and OMIM (https:// omim.org/) [21] were used to retrieve genes related to CI and establish disease gene data sets.

The UniProt database is used to correct the names of differential proteins and official gene symbols (Table S1, see supplementary materials). David Ver 6.8 was used for gene ontology (GO) annotation analysis and functional clustering analysis of differential proteins, edaravone potential, and CI genes [22]. The online tool STRING (http://www.string-db.org) was used for protein interaction analysis of differential proteins [23].

2.5. Detection of Oxidative Stress Indicators in Brain Tissue. After the brain tissue was homogenized with physiological saline, the contents of SOD, MDA, GSH, and NO were determined according to the instructions of the kit.

2.6. Detection of Serum AGTand CAT by ELISA. The serum of each group of rats was collected, placed in an anticoagulation tube, shaken, centrifuged $(3000 \mathrm{r} / \mathrm{min})$ for $15 \mathrm{~min}$, and the upper serum was collected. Serum AGT and CAT levels were detected by ELISA according to the instructions of the kit. Firstly, the AGT and CAT monoclonal antibodies are coated on the ELISA plate, and the standards and samples are added to make the AGT and CAT bound to the corresponding monoclonal antibodies. Biotinylated anti-rat AGT and CAT antibodies are added to form an immune complex and connect to the plate. Then, streptavidin labeled with horseradish peroxidase is combined with biotin, the enzyme substrate OPD is added, and after the yellow color appears, the stop solution sulfuric acid is added. The sample is detected by the microplate reader at a wavelength of $450 \mathrm{~nm}$ in accordance with the ELISA kit procedure. After the blank hole is zeroed, read the optical density (OD) value of each hole.

2.7. Statistical Analysis. The measurement data were expressed as mean \pm standard deviation (SD). The neurological function score (mNSS score) of the rats was analyzed by one-way analysis of variance using SPSS software 19.0. $P<0.05$ was considered to be statistically significant. 


\section{Results}

3.1. Neurological Score and Pathological Changes. The neurological function score of each model group was in the range of 1-3 points, which can indicate to a certain extent that the model is successful. The neurological function scores of the CN group and the CE group were lower than those of the CI group, indicating that the neurological function after drug intervention was better than the cerebral infarction model without drug intervention and to a certain extent that the drug has an intervention effect on CI (Table 1).

Under immunofluorescence, the BrdU signals appeared in the edaravone group, and it was considered that there were newborn nerve cells. The number of positive signals in the edaravone group was higher than that in the model group, indicating that the number of newborn nerve cells increased after drug treatment (Figure 1).

\subsection{Proteomics Analysis Results}

3.2.1. Differential Expressed Protein. A total of 1,340 proteins were identified in this study, of which 1,138 proteins contained quantitative information. With 1.3 times as the change threshold and $t$-test $P$ value $<0.05$ as the standard, then among the quantified proteins, the expression of 90 proteins in the $\mathrm{CI} / \mathrm{CN}$ comparison group was upregulated, and the expression of 26 proteins was downregulated (Table 2). In the CE/CI comparison group, the expression of 21 proteins was upregulated, and the expression of 41 proteins was downregulated (Table 3 ). The difference fold value change more than 1.5 times was regarded as a significant increase, and less than 0.77 was regarded as a significant decrease. There are overlapping proteins between CE/CI group and CI/CN group (Figure 2). They were considered to be the adjustable targets of edaravone after CI. The expression matrix of these proteins is shown in Figure 3.

\subsubsection{Proteomics Findings Validated by ELISA.} Compared with sham operation group, the AGT and CAT in model group were increased $(P<0.05)$. Compared with model group, the AGT and CAT in edaravone group were decreased $(P<0.05)$. This is consistent with the findings of proteomics (Figure 4$)$.

3.2.3. Bioinformatics Analysis. One hundred and fifty-three (153) differentially expressed proteins were introduced into String to construct PPI network (Figure 5) and subjected to enrichment analysis. The enrichment results show that these 153 differentially expressed proteins are related to 12 signaling pathways, 84 biological processes, 41 cell components, and 29 molecular functions. Their fold enrichment, $P$ value and count of each signaling pathway, biological process, cell component, and molecular function are shown in Figure 6. The biological processes is mainly related to response to muscle filament sliding, platelet degranulation,
TABLE 1: Neurological score.

\begin{tabular}{lcc}
\hline Group & Score $(X \pm S)$ & $P$ value \\
\hline CN & 0 & - \\
CI & $2.813 \pm 0.403$ & - \\
CE & $2.067 \pm 0.799$ & 0.0012 \\
\hline
\end{tabular}

muscle contraction, cellular oxidant detoxification, proteolysis, negative regulation of endopeptidase activity, response to drug, response to zinc ion, response to ethanol, protein refolding, retina homeostasis, response to unfolded protein, cell-cell adhesion, cardiac muscle contraction, response to hydrogen peroxide, skeletal muscle contraction, canonical glycolysis, defense response to fungus, response to selenium ion, and so on. The cell component is related to extracellular exosome, extracellular space, extracellular region, cytosol, extracellular matrix, blood microparticle, focal adhesion, muscle myosin complex, membrane, sarcomere, melanosome, stress fiber, platelet alpha granule lumen, actin filament, cell-cell adherens junction, myosin filament, $\mathrm{Z}$ disc, basement membrane, and so on. The molecular function is related to structural constituent of muscle, glycoprotein binding, identical protein binding, antioxidant activity, calcium ion binding, serine-type endopeptidase activity, actin binding, cadherin binding involved in cell-cell adhesion, MHC class II protein complex binding, protein binding, poly(A) RNA binding, unfolded protein binding, virion binding, endopeptidase inhibitor activity, actin filament binding, cytoskeletal protein binding, serine-type endopeptidase inhibitor activity, and so on. The signaling pathway is mainly related to metabolic pathways, biosynthesis of amino acids, glycolysis/gluconeogenesis, arginine biosynthesis, carbon metabolism, and so on (Figure 6). Their details were shown in Table S2.

\subsection{Chemoinformatics Analysis Results}

3.3.1. Edaravone-CI PPI Network. Edaravone target and CI gene were introduced into String to construct edaravone-CI PPI network. This network is composed of 141 nodes and 1324 edges. The average node degree is 18.8 , and the average local clustering coefficient is 0.526 (Figure 7).

3.3.2. Enrichment Analysis Results. The Edaravone target and CI gene were input into David for enrichment analysis and returns 14 CI-related signaling pathways, 63 biological processes, 21 cell components, and 21 molecular function. Their $P$ value, fold enrichment and count of each signaling pathway, biological process, cell component, and molecular function are shown in Figure 8. The biological process is related to blood coagulation, platelet activation, proteolysis, platelet degranulation, fibrinolysis, response to hypoxia, and so on. The cell component is related to extracellular space, extracellular region, platelet alpha granule lumen, cell surface, extracellular matrix, extracellular exosome, and so on. The molecular function is related to serine-type endopeptidase activity, protease binding, protein binding, heparin binding, glycoprotein binding, receptor binding, and so on. 


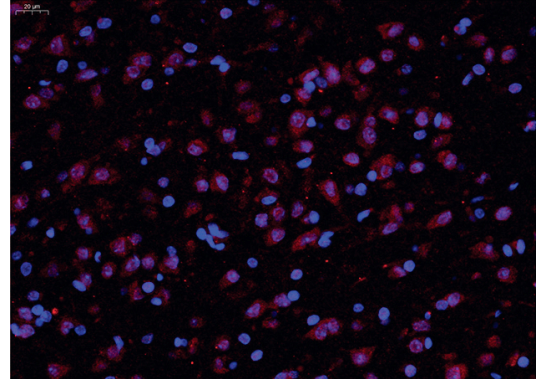

(a)

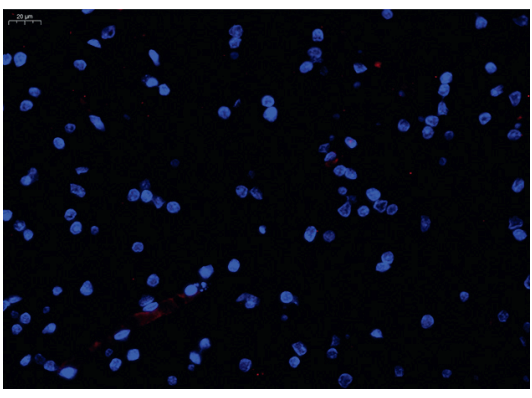

(b)

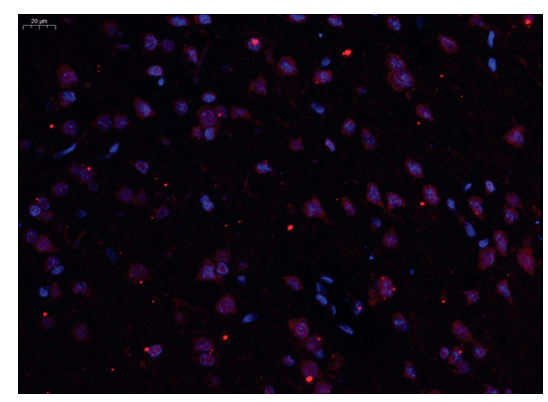

(c)

Figure 1: Pathological changes (400X). (a) Sham operation group. (b) Model group. (c) Edaravone group.

TABle 2: Differential expressed protein of $\mathrm{CI} / \mathrm{CN}$ group.

\begin{tabular}{|c|c|c|c|}
\hline Protein description & Gene name & $\mathrm{CI} / \mathrm{CN}$ ratio & Regulated type \\
\hline Endoplasmin & Hsp90b1 & 1.395 & $\mathrm{Up}$ \\
\hline 4-Trimethylaminobutyraldehyde dehydrogenase & Aldh9a1 & 1.67 & Up \\
\hline Betaine-homocysteine S-methyltransferase 1 & Bhmt & 4.005 & Up \\
\hline Creatine kinase M-type & Ckm & 2.532 & Up \\
\hline Calpastatin & Cast & 1.368 & $\mathrm{Up}$ \\
\hline Pyruvate kinase & Pkm & 1.667 & Up \\
\hline Protein IGHM & IGHM & 0.707 & Down \\
\hline COP9 signalosome complex subunit 1 & Gps1 & 1.65 & Up \\
\hline Transgelin & Tagln & 1.605 & Up \\
\hline Insulin-like growth factor I & Igf1 & 0.632 & Down \\
\hline Protein P4ha2 & P4ha2 & 1.457 & Up \\
\hline Polyadenylate-binding protein 1 & Pabpc1 & 1.544 & $\mathrm{Up}$ \\
\hline "6-Phosphofructo-2-kinase/fructose-2,6-bisphosphatase 1" & Pfkfb1 & 1.723 & Up \\
\hline Protein F5 & F5 & 0.692 & Down \\
\hline Protein Myh1 & Myh1 & 2.155 & Up \\
\hline Protein Ugp2 & Ugp2 & 1.635 & $\mathrm{Up}$ \\
\hline Protein LOC103691744 & LOC103691744 & 4.174 & Up \\
\hline Protein Myom1 & Myom1 & 1.745 & Up \\
\hline Protein RGD1564614 & RGD1564614 & 0.711 & Down \\
\hline Protein LOC299282 & Serpina3n & 1.632 & Up \\
\hline Protein S100-A9 & S100a9 & 1.873 & $\mathrm{Up}$ \\
\hline Haptoglobin & $\mathrm{Hp}$ & 1.9 & Up \\
\hline Filamin-C & Flnc & 1.991 & Up \\
\hline "Ubiquilin 1 , isoform CRA_a" & Ubqln 1 & 1.658 & $\mathrm{Up}$ \\
\hline Myl9 protein & Myl9 & 1.792 & $\mathrm{Up}$ \\
\hline Filamin alpha & Flna & 1.81 & $\mathrm{Up}$ \\
\hline Matrix metalloproteinase 19 & Mmp19 & 0.724 & Down \\
\hline Glycerol kinase & Gk & 2.08 & Up \\
\hline Protein papln & Papln & 0.734 & Down \\
\hline Calponin & Cnn2 & 0.714 & Down \\
\hline Protein aldh8a1 & Aldh8a1 & 1.784 & $\mathrm{Up}$ \\
\hline Neutrophilic granule protein (predicted) & Ngp & 1.729 & Up \\
\hline Protein col14al & Coll4a1 & 2.295 & $\mathrm{Up}$ \\
\hline Protein Atp6vla & Atp6v1a & 1.341 & Up \\
\hline Protein LOC100362751 & LOC498555 & 2.379 & $\mathrm{Up}$ \\
\hline Leukocyte cell-derived chemotaxin 2 (predicted) & Lect2 & 0.739 & Down \\
\hline Protein Rsu1 & Rsu1 & 0.675 & Down \\
\hline "Fructose-1,6-bisphosphatase 1" & Fbp1 & 1.709 & Up \\
\hline Heat shock $70 \mathrm{kDa}$ protein 4 & Hspa4 & 1.526 & Up \\
\hline Protein Myh1 & Myh1 & 1.987 & Up \\
\hline S-adenosylmethionine synthase & Matla & 1.787 & Up \\
\hline Protein Igkv8-27 & Igkv8-27 & 0.536 & Down \\
\hline Protein Lama2 & Lama2 & 1.429 & $\mathrm{Up}$ \\
\hline Protein Rrbp1 & Rrbp1 & 2.254 & Up \\
\hline
\end{tabular}


TABle 2: Continued.

\begin{tabular}{|c|c|c|c|}
\hline Protein description & Gene name & $\mathrm{CI} / \mathrm{CN}$ ratio & Regulated type \\
\hline Protein Lamc1 & Lamc1 & 2.232 & $\mathrm{Up}$ \\
\hline "Nitrilase 1, isoform CRA_a" & Nit1 & 1.789 & Up \\
\hline Protein Myh1 & Myh2 & 2.763 & $\mathrm{Up}$ \\
\hline Myomesin 2 & Myom2 & 2.09 & Up \\
\hline "ATPase, $\mathrm{H}+$ transporting, $\mathrm{V} 1$ subunit $\mathrm{E}$ isoform 1, isoform CRA_a" & Atp6v1e1 & 1.351 & $\mathrm{Up}$ \\
\hline Lamin-B1 & Lmnb1 & 2.227 & Up \\
\hline "Prolactin induced protein, isoform CRA_d" & Pip & 2.152 & $\mathrm{Up}$ \\
\hline Myosin-7 & Myh7 & 2.873 & $\mathrm{Up}$ \\
\hline "Lamin A, isoform CRA_b" & Lmna & 2.146 & $\mathrm{Up}$ \\
\hline Integrin alpha $\mathrm{M}$ & Itgam & 1.393 & $\mathrm{Up}$ \\
\hline "RCG39455, isoform CRA_a" & Tsku & 0.602 & Down \\
\hline Acylamino-acid-releasing enzyme & Apeh & 0.65 & Down \\
\hline ATP-citrate synthase & Acly & 1.686 & Up \\
\hline Protein Sec24d & Sec24d & 1.551 & $\mathrm{Up}$ \\
\hline "Protein S (alpha), isoform CRA_b" & Pros1 & 0.679 & Down \\
\hline 40 S ribosomal protein $\mathrm{S} 6$ & Rps6 & 0.535 & Down \\
\hline Ras-related protein Rab-11B & Rab11b & 1.566 & Up \\
\hline Bifunctional purine biosynthesis protein PURH & Atic & 1.777 & Up \\
\hline Ribonuclease 4 & Rnase4 & 0.762 & Down \\
\hline Anionic trypsin 1 & Prss 1 & 4.631 & Up \\
\hline Angiotensinogen & Agt & 1.777 & $\mathrm{Up}$ \\
\hline "Myosin light chain $1 / 3$, skeletal muscle isoform" & Myl1 & 2.052 & $\mathrm{Up}$ \\
\hline "Myosin regulatory light chain 2 , skeletal muscle isoform" & Mylpf & 2.39 & $\mathrm{Up}$ \\
\hline Tropomyosin alpha-1 chain & Tpm1 & 2.41 & Up \\
\hline Catalase & Cat & 1.669 & $\mathrm{Up}$ \\
\hline Protein disulfide-isomerase & P4hb & 1.408 & Up \\
\hline Fructose-bisphosphate aldolase A & Aldoa & 1.927 & $\mathrm{Up}$ \\
\hline Elongation factor 2 & Eef2 & 1.652 & $\mathrm{Up}$ \\
\hline Delta-aminolevulinic acid dehydratase & Alad & 1.858 & $\mathrm{Up}$ \\
\hline Alcohol dehydrogenase 1 & Adh1 & 2.348 & Up \\
\hline "Carbamoyl-phosphate synthase [ammonia], mitochondrial" & Cps1 & 4.184 & $\mathrm{Up}$ \\
\hline Tropomyosin alpha-4 chain & Tpm4 & 0.647 & Down \\
\hline "Glycogen phosphorylase, liver form" & Pygl & 1.704 & $\mathrm{Up}$ \\
\hline Adenosylhomocysteinase & Ahcy & 1.729 & $\mathrm{Up}$ \\
\hline Galectin-1 & Lgals1 & 0.599 & Down \\
\hline Serotransferrin & Tf & 1.471 & Up \\
\hline cAMP-dependent protein kinase type II-beta regulatory subunit & Prkar2b & 0.506 & Down \\
\hline Insulin-like growth factor-binding protein 2 & Igfbp2 & 0.661 & Down \\
\hline "Phospholipase A2, membrane associated" & Pla2g2a & 0.562 & Down \\
\hline Phosphoglycerate kinase 1 & Pgk1 & 1.581 & Up \\
\hline $60 S$ acidic ribosomal protein $\mathrm{P} 0$ & Rplp0 & 2.136 & $\mathrm{Up}$ \\
\hline Insulin-like growth factor-binding protein 1 & Igfbp1 & 0.56 & Down \\
\hline Cytosolic 10-formyltetrahydrofolate dehydrogenase & Aldh1l1 & 1.968 & $\mathrm{Up}$ \\
\hline Protein S100-A8 & S100a8 & 1.535 & $\mathrm{Up}$ \\
\hline Ficolin-2 & $\mathrm{Fcn} 2$ & 1.54 & $\mathrm{Up}$ \\
\hline Tropomyosin beta chain & Tpm2 & 3.257 & $\mathrm{Up}$ \\
\hline 14-3-3 protein epsilon & Ywhae & 1.607 & $\mathrm{Up}$ \\
\hline Heat shock cognate $71 \mathrm{kDa}$ protein & Hspa8 & 2.161 & $\mathrm{Up}$ \\
\hline "60 kDa heat shock protein, mitochondrial" & Hspd 1 & 1.749 & Up \\
\hline "Actin, alpha cardiac muscle $1 "$ & Actc1 & 1.579 & $\mathrm{Up}$ \\
\hline "Actin, alpha skeletal muscle" & Actal & 3.221 & $\mathrm{Up}$ \\
\hline Heat shock protein HSP 90 -alpha & Hsp90aa1 & 1.606 & $\mathrm{Up}$ \\
\hline Pleckstrin & Plek & 0.638 & Down \\
\hline Interleukin-1 receptor-associated kinase-like 2 & Irak2 & 0.447 & Down \\
\hline Argininosuccinate lyase & Asl & 1.607 & $\mathrm{Up}$ \\
\hline Aspartyl aminopeptidase & Dnpep & 1.473 & $\mathrm{Up}$ \\
\hline Tubulin alpha- $4 \mathrm{~A}$ chain & Tuba4a & 0.683 & Down \\
\hline Ras-related protein Rap-1b & Raplb & 0.691 & Down \\
\hline Secreted phosphoprotein 24 & Spp2 & 0.521 & Down \\
\hline Nucleobindin-1 & Nucb1 & 1.625 & Up \\
\hline
\end{tabular}


TABle 2: Continued.

\begin{tabular}{|c|c|c|c|}
\hline Protein description & Gene name & $\mathrm{CI} / \mathrm{CN}$ ratio & Regulated type \\
\hline Receptor-type tyrosine-protein phosphatase F & Ptprf & 1.443 & Up \\
\hline COP9 signalosome complex subunit 4 & Cops 4 & 1.441 & Up \\
\hline COP9 signalosome complex subunit 3 & Cops3 & 1.787 & Up \\
\hline Group specific component & Gc & 2.016 & Up \\
\hline Carboxypeptidase & Ctsa & 1.415 & Up \\
\hline Phenylalanine hydroxylase & Pah & 2.261 & Up \\
\hline Nucleotide exchange factor SIL1 & Sil1 & 1.666 & Up \\
\hline Desmin & Des & 2.285 & Up \\
\hline Alpha-2 antiplasmin & Serpinf1 & 1.488 & Up \\
\hline Aflatoxin $\mathrm{B} 1$ aldehyde reductase member 2 & Akr7a2 & 1.647 & Up \\
\hline "Tropomyosin 1, alpha, isoform CRA_a" & Tpm1 & 3.376 & $\mathrm{Up}$ \\
\hline Hepcidin & Hamp & 1.643 & Up \\
\hline Guanine deaminase & Gda & 1.566 & $\mathrm{Up}$ \\
\hline Peroxiredoxin- 4 & $\operatorname{Prdx} 4$ & 1.601 & Up \\
\hline
\end{tabular}

TABLE 3: Differential expressed protein of CE/CI group.

\begin{tabular}{|c|c|c|c|}
\hline Protein description & Gene name & $\mathrm{CE} / \mathrm{CI}$ ratio & Regulated type \\
\hline 4-Trimethylaminobutyraldehyde dehydrogenase & Aldh9a1 & 0.739 & Down \\
\hline Betaine-homocysteine S-methyltransferase 1 & Bhmt & 0.42 & Down \\
\hline Creatine kinase M-type & $\mathrm{Ckm}$ & 0.637 & Down \\
\hline Galectin & Lgals3 & 1.564 & Up \\
\hline Thyroglobulin & $\operatorname{Tg}$ & 2.708 & $\mathrm{Up}$ \\
\hline "6-Phosphofructo-2-kinase/fructose-2,6-bisphosphatase 1" & Pfkfb1 & 0.62 & Down \\
\hline Complement factor I & Cfi & 0.574 & Down \\
\hline Protein TNC & TNC & 1.606 & $\mathrm{Up}$ \\
\hline Peptidyl-prolyl cis-trans isomerase & Ppia & 0.729 & Down \\
\hline Protein F11 & F11 & 1.337 & $\mathrm{Up}$ \\
\hline Protein LOC103691744 & LOC103691744 & 0.204 & Down \\
\hline Protein RGD1310507 & RGD1310507 & 0.697 & Down \\
\hline Histidine-rich glycoprotein & Hrg & 5.24 & $\mathrm{Up}$ \\
\hline Coagulation factor XII & F12 & 0.669 & Down \\
\hline LOC683667 protein & Sri & 1.321 & $\mathrm{Up}$ \\
\hline Glycerol kinase & Gk & 0.537 & Down \\
\hline Protein Fat4 & Fat4 & 1.45 & $\mathrm{Up}$ \\
\hline Carboxylic ester hydrolase & Ceslc & 0.421 & Down \\
\hline RCG21066 & rCG_21066 & 0.686 & Down \\
\hline Protein IGHM & IGHM & 1.411 & Up \\
\hline "Sushi, Von Willebrand factor type A, EGF and pentraxin domain-containing protein 1" & Svep1 & 1.454 & $\mathrm{Up}$ \\
\hline "Procollagen, type XVIII, alpha 1, isoform CRA_a" & Coll8a1 & 1.499 & Up \\
\hline Serine protease inhibitor $\mathrm{A} 3 \mathrm{M}$ & Serpina3m & 0.616 & Down \\
\hline "Fructose-1,6-bisphosphatase 1" & Fbp1 & 0.602 & Down \\
\hline Serine protease hepsin & Hpn & 1.413 & Up \\
\hline S-adenosylmethionine synthase & Matla & 0.521 & Down \\
\hline "Troponin I, fast skeletal muscle" & Tnni2 & 0.722 & Down \\
\hline "Fibulin 2, isoform CRA_a" & Fbln 2 & 1.354 & $\mathrm{Up}$ \\
\hline Apolipoprotein C-II (predicted) & Apoc2 & 2.066 & $\mathrm{Up}$ \\
\hline Heat shock $27 \mathrm{kDa}$ protein 1 & Hspb1 & 1.671 & Up \\
\hline "Protein S (alpha), isoform CRA_b" & Pros1 & 1.448 & $\mathrm{Up}$ \\
\hline Protein LOC691828 & LOC691828 & 0.718 & Down \\
\hline Glutathione peroxidase & Gpx1 & 0.399 & Down \\
\hline 40 S ribosomal protein S6 & Rps6 & 2.075 & Up \\
\hline Peroxiredoxin- 6 & Prdx6 & 0.582 & Down \\
\hline Lysozyme C-1 & Lyz1 & 0.675 & Down \\
\hline Anionic trypsin 1 & Prss1 & 0.272 & Down \\
\hline Angiotensinogen & Agt & 0.687 & Down \\
\hline Serum albumin & Alb & 0.67 & Down \\
\hline Catalase & Cat & 0.658 & Down \\
\hline
\end{tabular}


TABLE 3: Continued.

\begin{tabular}{|c|c|c|c|}
\hline Protein description & Gene name & $\mathrm{CE} / \mathrm{CI}$ ratio & Regulated type \\
\hline Alcohol dehydrogenase 1 & Adh1 & 0.442 & Down \\
\hline Beta-2-microglobulin & $\mathrm{B} 2 \mathrm{~m}$ & 0.656 & Down \\
\hline "Carbamoyl-phosphate synthase [ammonia], mitochondrial" & Cps1 & 0.297 & Down \\
\hline Galectin-1 & Lgals1 & 1.658 & Up \\
\hline Cysteine-rich secretory protein 1 & Crisp1 & 0.439 & Down \\
\hline Serotransferrin & Tf & 0.471 & Down \\
\hline Cystatin-C & Cst3 & 0.625 & Down \\
\hline Carbonic anhydrase 2 & $\mathrm{Ca} 2$ & 0.533 & Down \\
\hline Cytosolic 10-formyltetrahydrofolate dehydrogenase & Aldh1l1 & 0.599 & Down \\
\hline C-reactive protein & Crp & 1.454 & $\mathrm{Up}$ \\
\hline Cadherin-17 & Cdh17 & 1.512 & $\mathrm{Up}$ \\
\hline Ficolin-2 & Fcn 2 & 0.724 & Down \\
\hline Interleukin-1 receptor-associated kinase-like 2 & Irak2 & 0.556 & Down \\
\hline Argininosuccinate lyase & Asl & 0.707 & Down \\
\hline Apolipoprotein $\mathrm{H}$ & Apoh & 0.644 & Down \\
\hline Protein Serpina4 & Serpina4 & 0.727 & Down \\
\hline Protein Hbb-b1 & LOC103694855 & 0.507 & Down \\
\hline Secreted phosphoprotein 24 & Spp2 & 1.849 & $\mathrm{Up}$ \\
\hline BPI fold-containing family a member 2 & Bpifa2 & 0.46 & Down \\
\hline Phenylalanine hydroxylase & Pah & 0.491 & Down \\
\hline Cathepsin D & Ctsd & 1.466 & Up \\
\hline Alpha-2 antiplasmin & Serpinf1 & 0.713 & Down \\
\hline
\end{tabular}

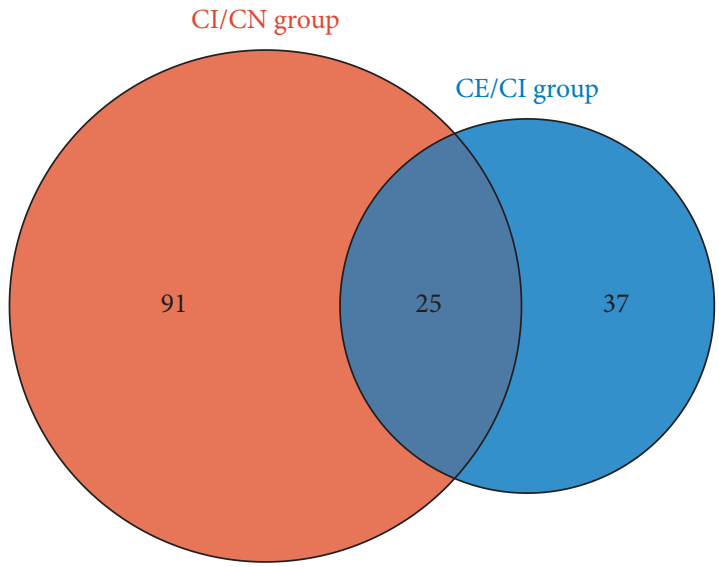

FIgURE 2: Venn diagram of CE/CI group and CI/CN group.

The signaling pathway is related to complement and coagulation cascades, platelet activation, TNF signaling pathway, insulin resistance, HIF-1 signaling pathway, FoxO signaling pathway, and so on (Figure 8). Their details were shown in Table S3.

3.4. Expression of Differentially Expressed Proteins in Brain Tissue. The expression of DEPs in each organ in each comparison group was analyzed, and the obtained DEPs were analyzed by Expression Atlas. After the tissue specificity is set to "brain," the protein with the number 10 in descending order or the protein with number $\geqq 10$ is matched. The brain tissue specificity of these proteins is ranked according to their expression strength in brain tissue and other tissues, and the detailed information is listed in Tables 4 and 5 .
Different proteins with upregulated expression and high brain specificity in the $\mathrm{CI} / \mathrm{CE}$ comparison group are cathepsin D, LOC683667 protein, galectin-1, C-reactive protein, and 40 S ribosomal protein S6.

Different proteins with downregulated expression and high brain specificity in the $\mathrm{CI} / \mathrm{CN}$ comparison group are cystatin-C, serotransferrin, peptidyl-prolyl cis-trans isomerase, beta-2-microglobulin, carbonic anhydrase2, angiotensinogen, peroxiredoxin-6, glutathione peroxidase, 4trimethylaminobutyraldehyde dehydrogenase, and catalase.

3.5. Oxidative Stress Indicators in Brain Tissue. Compared with the sham operation group, the MDA content of the model group was significantly increased, while the SOD and GSH content were significantly decreased $(P<0.05)$, indicating that the model was successful. Compared with the model group, the MDA content of the edaravone administration group significantly decreased, and the SOD and GSH content significantly increased $(P<0.05)$, indicating that edaravone can improve the brain SOD, MDA, and GSH content of rats (Figure 9).

\section{Discussion}

CI is a cerebrovascular disease characterized by brain blood supply disorder, local avascular necrosis, or softening of brain tissue caused by ischemia and hypoxia. Among them, neuronal programmed death (apoptosis, autophagy, iron death, scorch death, etc.) and necrosis exist simultaneously. The main pathological mechanisms include energy exhaustion, excitotoxicity, oxidative stress, endoplasmic reticulum dysfunction, and a large amount of immune cell infiltration and aggregation. Various cytokines are involved in a series of "cascade-like" cascade events, such as 


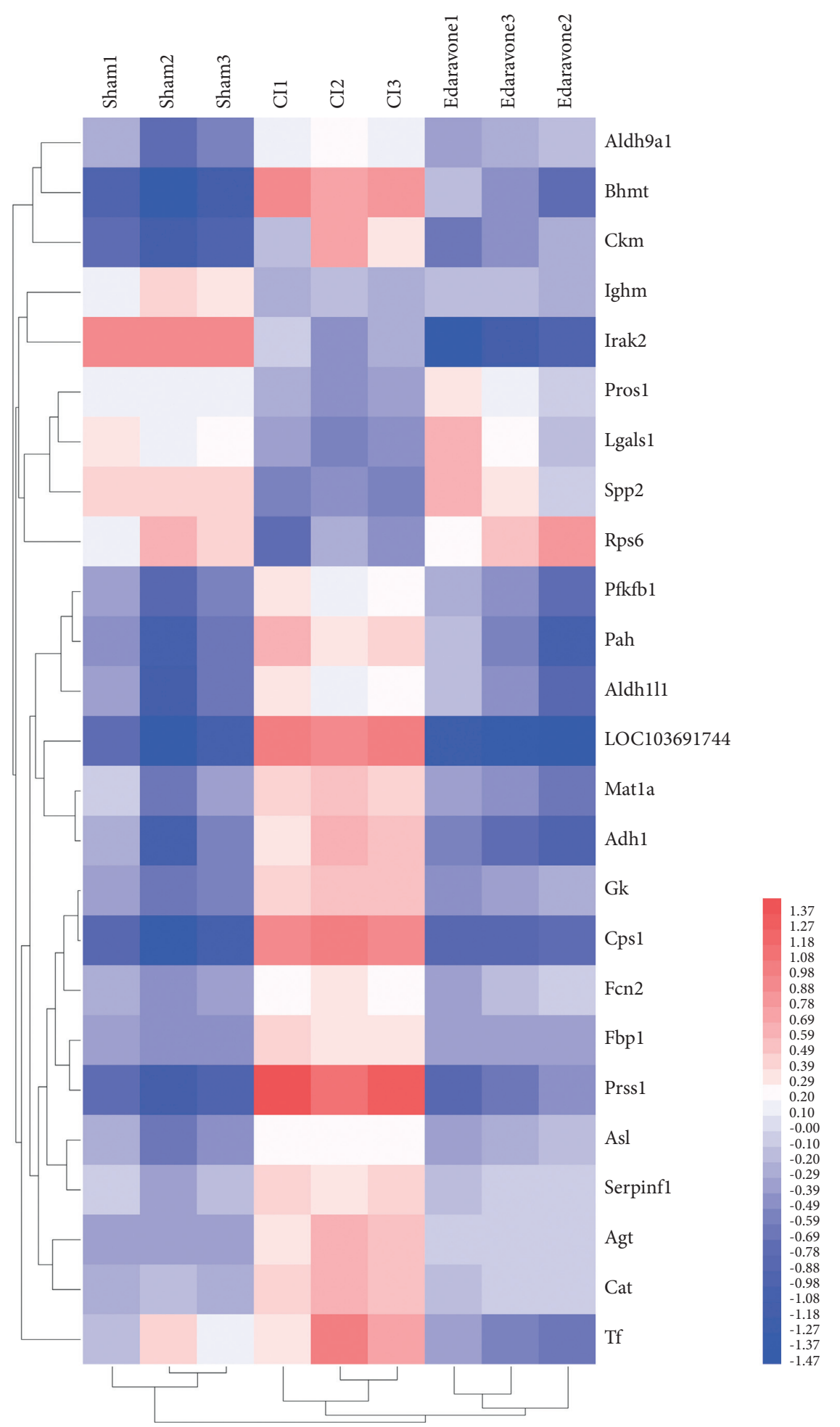

Figure 3: The expression matrix the 26 proteins.

neuroinflammation in the infarct area, which ultimately leads to the death of nerve cells. The damage caused by free radicals induced by oxidative stress is considered to be an important pathological basis for brain neuron damage in the onset of stroke. The chain reaction of free radicals causes excessive oxidation of cell membrane lipids and nuclear proteins and damages the structure and function of mitochondria, resulting in insufficient cell energy production, leading to cell death or apoptosis. Therefore, antioxidative stress response and scavenging excess free radicals may be important strategies for early CI to protect neurons and reduce damage.

Edaravone, as a free radical scavenger, has the effects of antioxidative stress and inhibiting lipid peroxidation. 

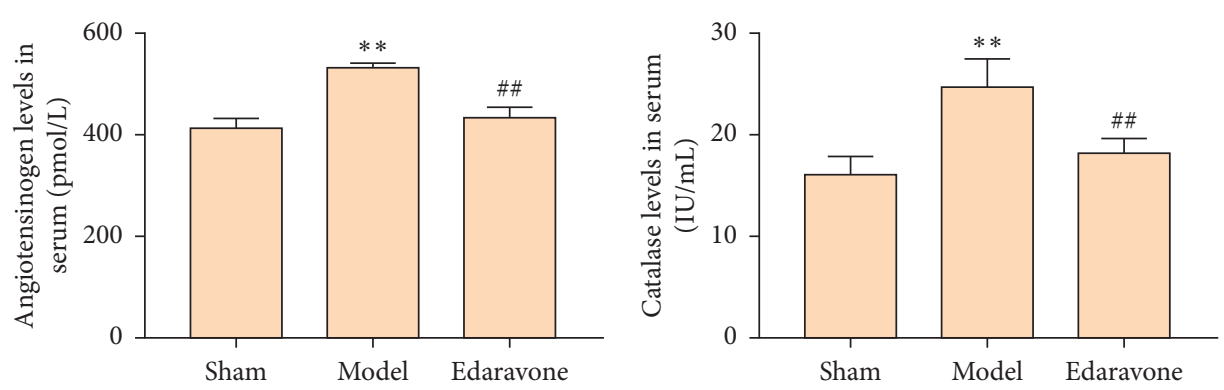

Figure 4: Serum AGT and CAT level (** compared with sham operation group, $P<0.05 ;{ }^{\# \#}$ compared with model group, $P<0.05$ ).

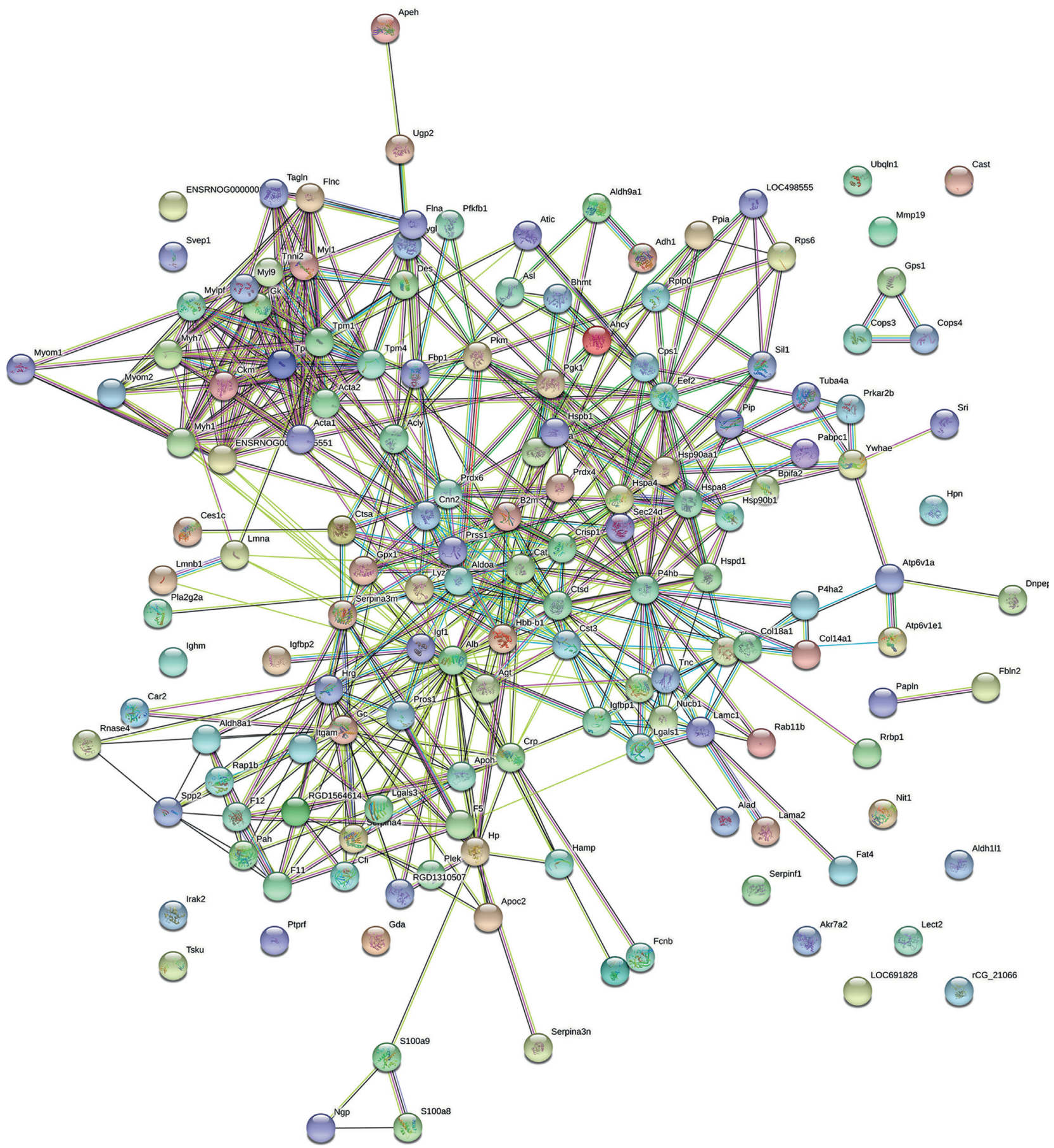

Figure 5: The PPI network of differentially expressed proteins. 


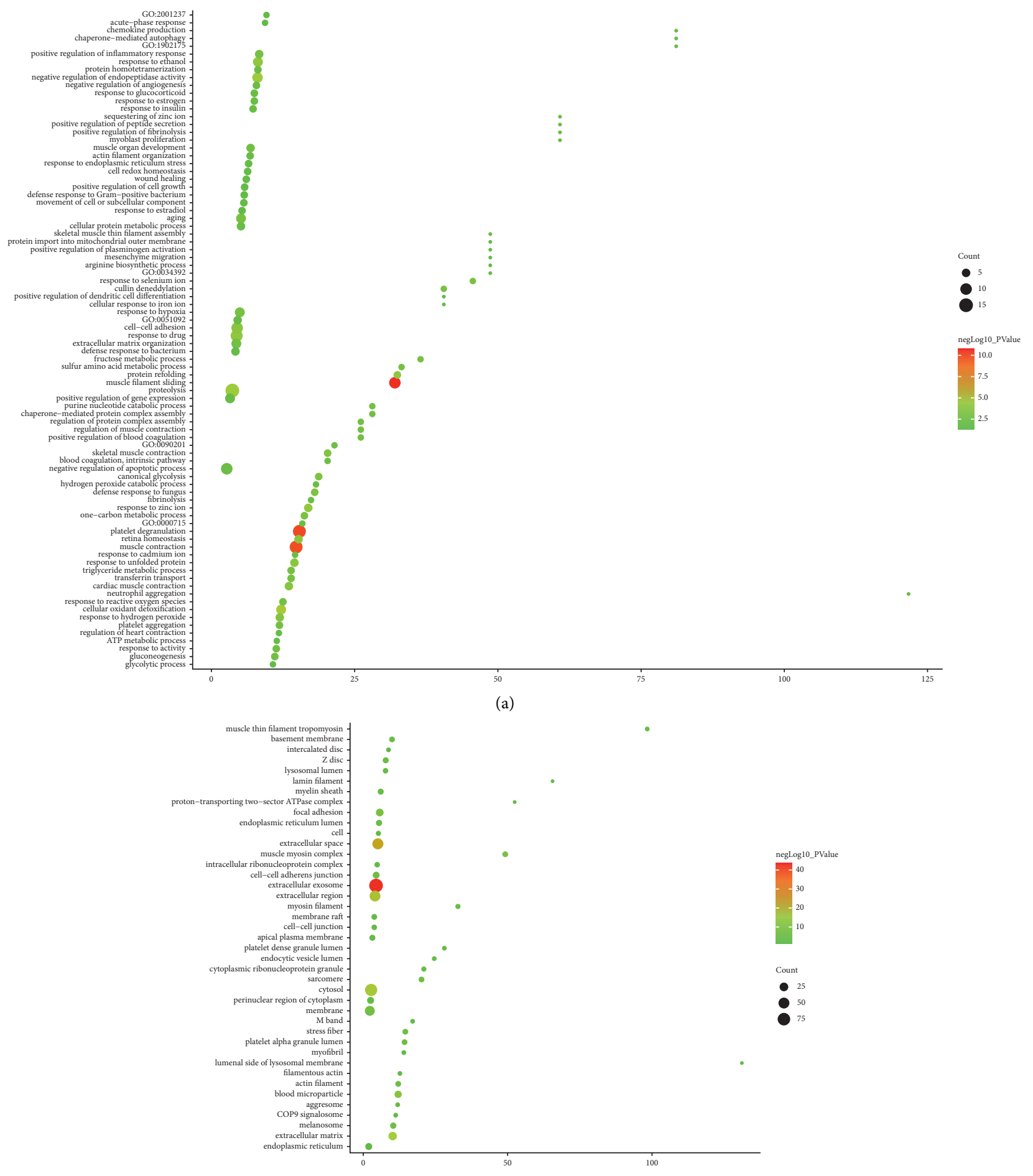

(b)

Figure 6: Continued. 


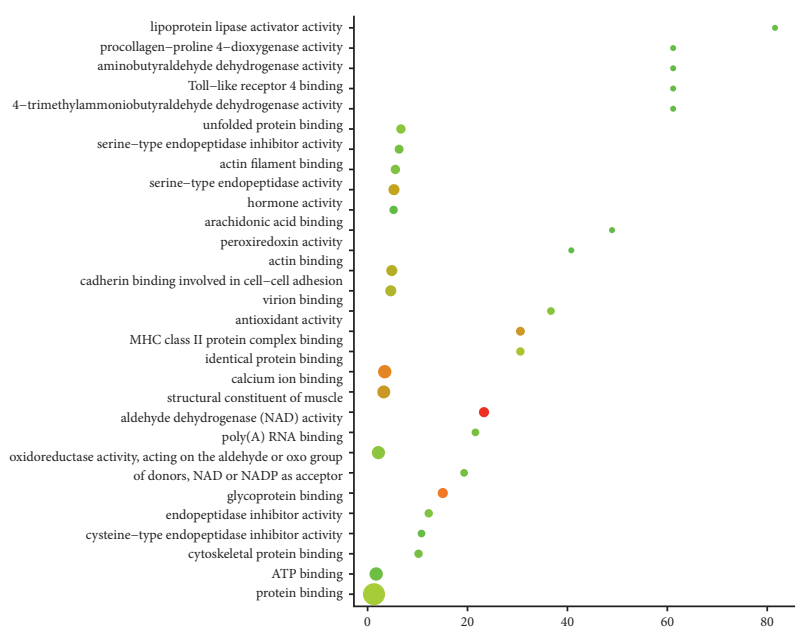

(c)
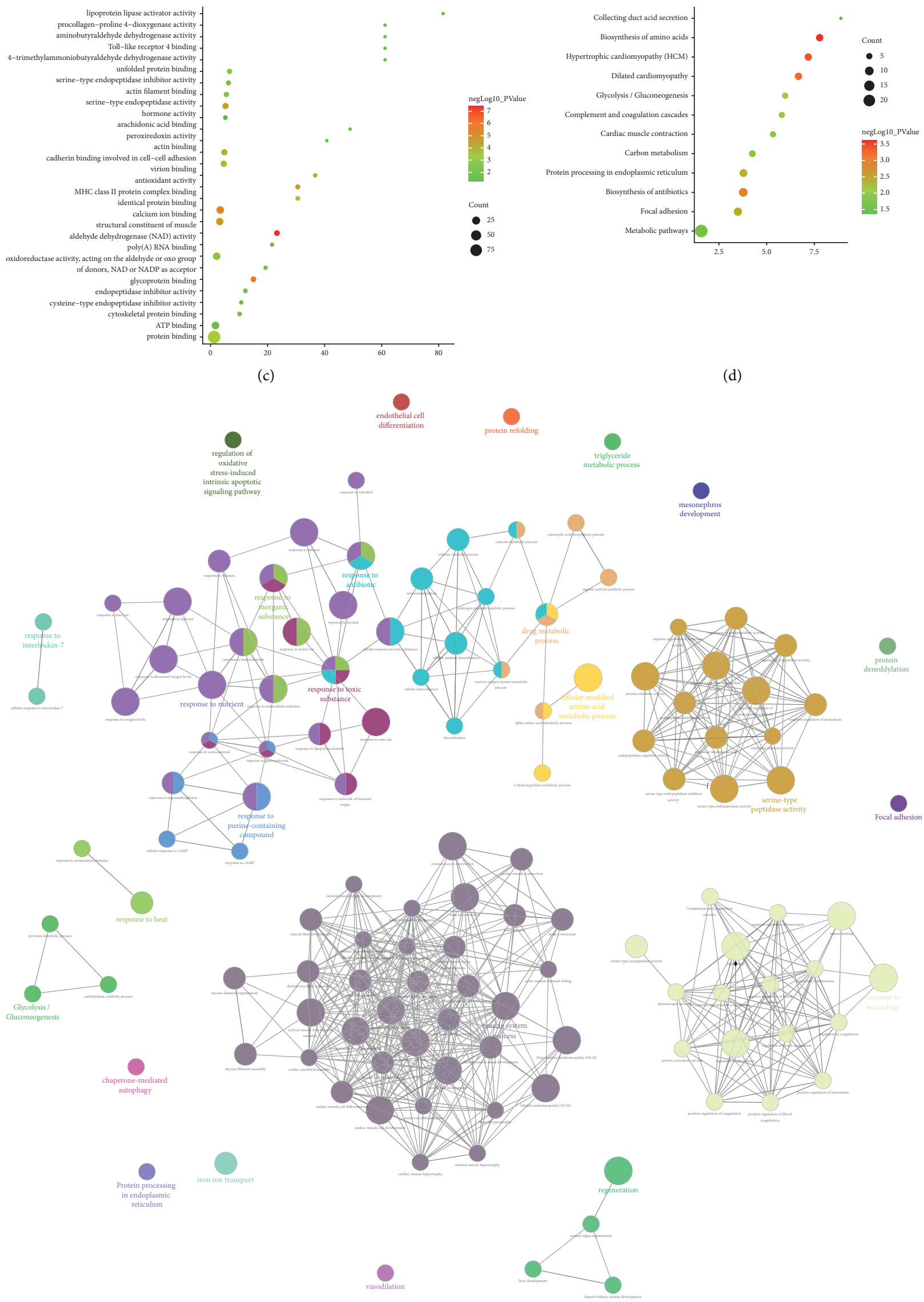

(e)

Figure 6: Continued. 


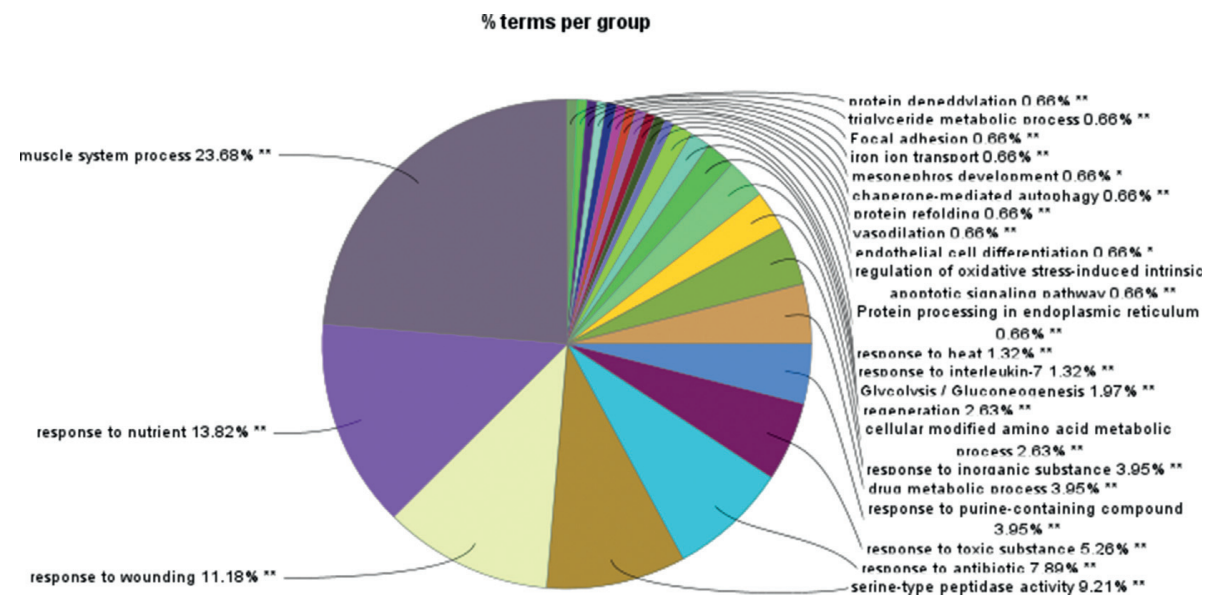

(f)

Figure 6: The results of enrichment analysis (a: biological processes; b: cell components; c molecular function; $d$ signaling pathways; $X$-axis stands for fold enrichment. e and f Cluego analysis).

Studies have confirmed that edaravone can protect body tissues by inhibiting the activity of cyclooxygenase (COX, including COX-1, and COX-2). COX-2 is mainly expressed in hippocampal tissue and cortical neurons and can mediate neuronal damage. COX-2 is a key enzyme and rate-limiting enzyme produced by prostaglandin E2 (PGE2) metabolism, and it can also promote the production of nitric oxide (NO), while PGE2 and NO can participate in various pathological processes, such as inflammation, oxidative stress, and apoptosis. Current clinical and basic research shows that edaravone, which has an antioxidant effect, may become a new type of drug for the treatment of CI. This study established the CI rat model and used serum proteomics to determine the serum markers for edaravone to interfere with CI. 153 differentially expressed proteins include Rps6, Spp2, Lgals1, Pros1, Aldh9a1, Fcn2, Serpinf1, Asl, Agt, Cat, Ckm, Pfkfb1, Fbp1, Aldh1l1, Irak2, Gk, Mat1a, Pah, Tf, Adh1, Bhmt, Cps1, Prss1, LOC103691744, Tpm1, and Myh1. Recent studies have shown that ribosomal protein S6 (rpS6), extracellular signal-regulated mitogen-activated protein (MAP) kinase p44/42 (p44/42MAPK), and ribosomal protein S6 kinase (S6K) are significantly reduced in the brains of hibernating squirrels and rats. Therefore, we believe that the downregulation of rpS6 signal transduction may be an important reason for the increased cell tolerance to cerebral ischemia observed during hibernation numbness and after ischemic preconditioning, by inhibiting protein synthesis and/or energy expenditure [24]. Qu et al. showed that galectin-1 (Gal-1) can regulate the proliferation of a variety of cells and play an important role after nervous system injury. Galectin-1 can reduce astrocyte damage and improve the recovery of rats with focal cerebral ischemia [25]. Ishibashi S. et al. also found that galectin-1 regulates neurogenesis in the subventricular zone and promotes functional recovery after stroke [26]. Agt is the gene encoding angiotensinogen, and current studies have shown that its polymorphism is closely related to stroke, especially cerebral small vessel disease [27-29]. Current research shows that
Catalase (CAT) has important physiological functions in oxidative stress. CAT can quickly remove the toxic substances produced by cell metabolism, so as to protect sulfhydrylase and membrane protein and detoxify together with glutathione peroxidase (GSHPx). It plays an important protective role against cerebral ischemia in CI patients $[30,31]$. Aldh1l1 is mainly related to the proliferation of astrocytes after CI [32]. Interleukin-1 receptor-associated kinases (IRAKs) are mainly related to inflammation and cell apoptosis after cerebral ischemia and reperfusion [33].

The enrichment analysis of these 153 differentially expressed proteins involved a total of 12 signal pathways, 84 biological processes, 41 cell components, and 29 molecular functions. The biological process is mainly in platelet activation and aggregation (GO:0002576 platelet degranulation, GO:0070527 platelet aggregation), oxidative stress (GO:0098869 cellular oxidant detoxification; GO: 0006986 response to unfolded protein; GO: 0042542 response to hydrogen peroxide; GO: 0000302 response to reactive oxygen species; GO: 0090201 negative regulation of release of cytochrome $c$ from mitochondria; GO:0042744 hydrogen peroxide catabolic process; GO:0045454 cell redox homeostasis), intercellular adhesion (GO:0098609 cell-cell adhesion), glycolysis and gluconeogenesis (GO:0061621 canonical glycolysis; GO:0006094 gluconeogenesis), iron metabolism (GO:0033572 transferrin transport; GO:0071281 cellular response to iron ion), hypoxia (GO:0001666 response to hypoxia), inflammatory chemokines and their mediated signal transduction (GO:0050729 positive regulation of inflammatory response; GO:0051092 positive regulation of NF-kappaB transcription factor activity; GO: 0032602 chemokine production) reaction, coagulation (GO:0030194 positive regulation of blood coagulation; GO: 0007597 blood coagulation, intrinsic pathway), apoptosis (GO:0043066 negative regulation of apoptotic process; GO: 1902175 regulation of oxidative stress-induced intrinsic apoptotic signaling pathway), fibrinolysis system (GO: 


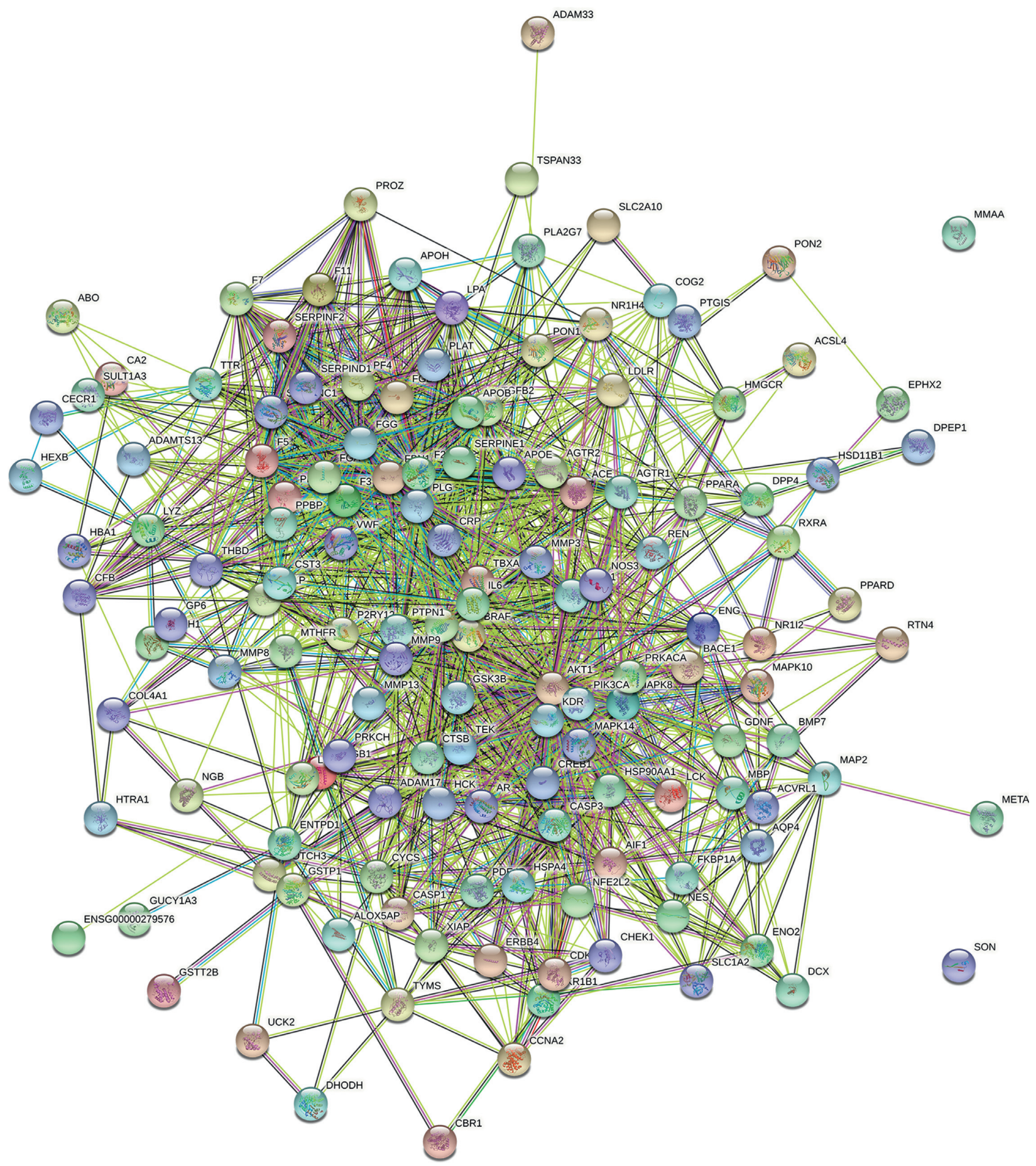

Figure 7: Edaravone-CI PPI network.

0042730 fibrinolysis; GO:0051919 positive regulation of fibrinolysis); angiogenesis (GO:0016525 negative regulation of angiogenesis) endoplasmic reticulum stress (GO: 0034976 response to endoplasmic reticulum stress), and energy metabolism (GO:0046034 ATP metabolic process; GO:0045040 protein import into mitochondrial outer membrane). These biological modules play an important role in the acute pathological process of cerebral infarction.
The molecular functions also focused on oxidative stress (GO:0016209 antioxidant activity; GO:0004029 aldehyde dehydrogenase (NAD) activity; GO: 0016620 oxidoreductase activity, acting on the aldehyde or oxo group of donors, NAD or NADP as acceptor), calcium ion binding (GO:0005509 calcium ion binding; GO: 0098641 cadherin binding involved in cell-cell adhesion), energy metabolism (GO:0005524 ATP binding), 


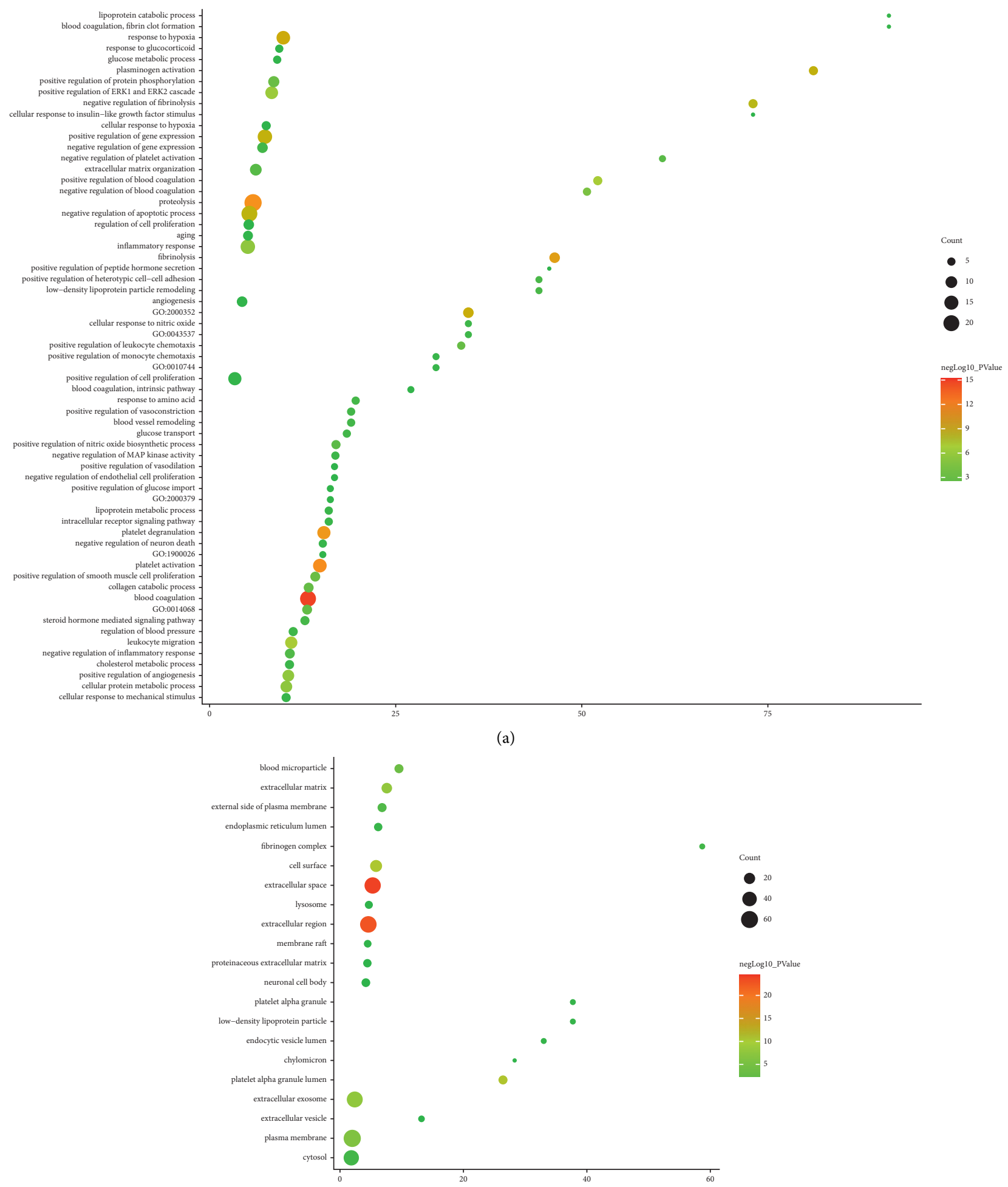

(b)

Figure 8: Continued. 


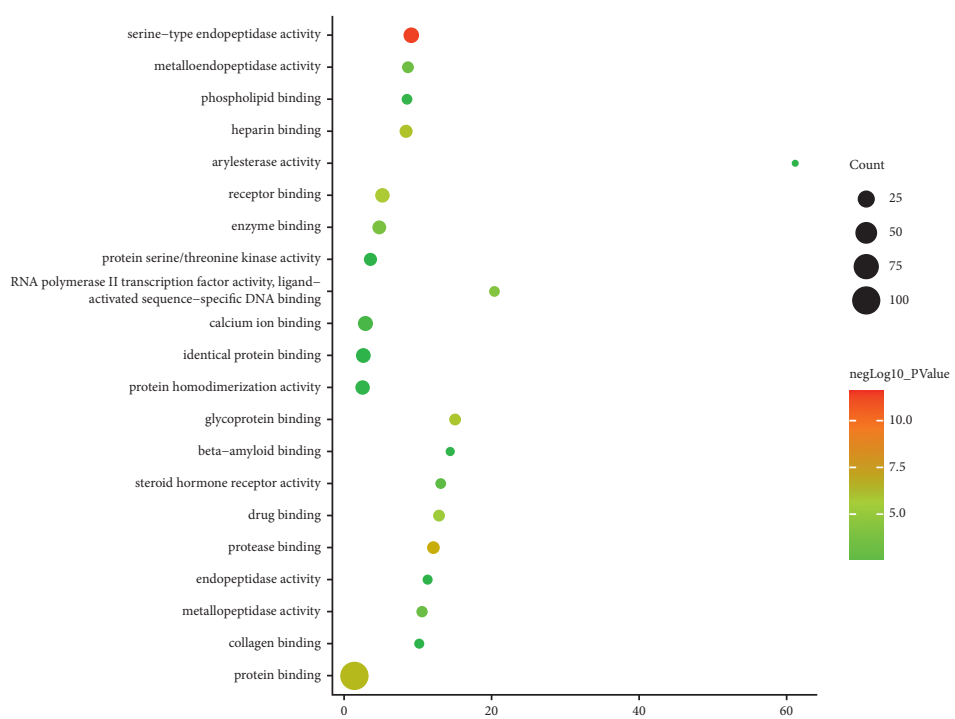

(c)

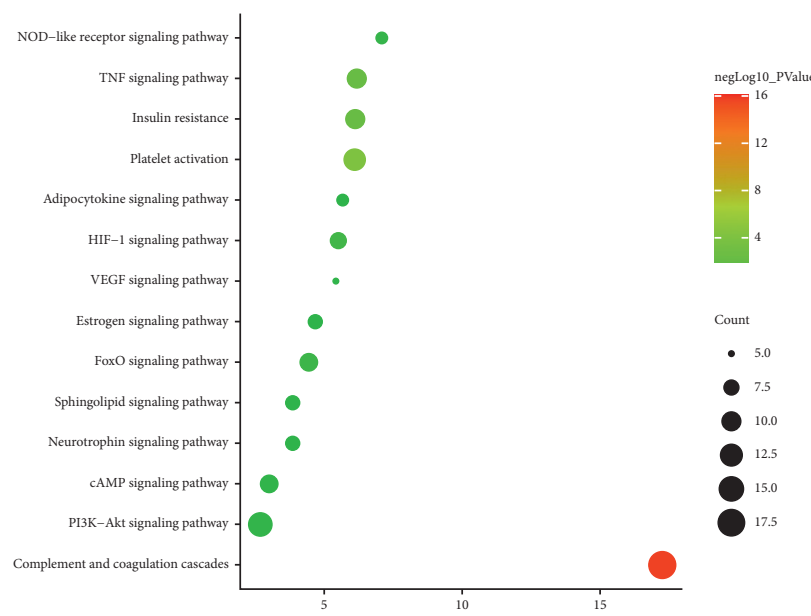

(d)

Figure 8: Continued. 


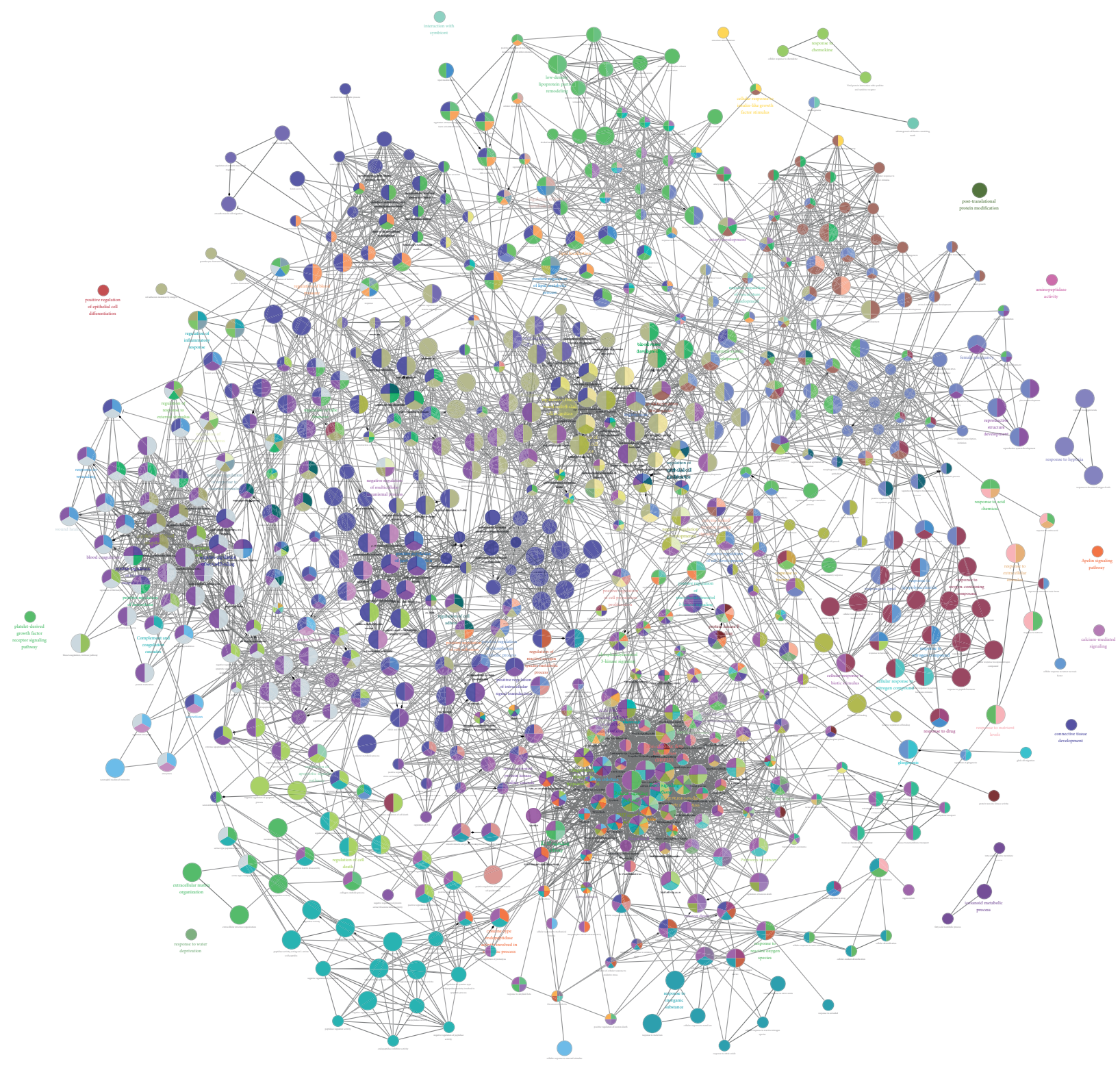

(e)

$\%$ terms per group

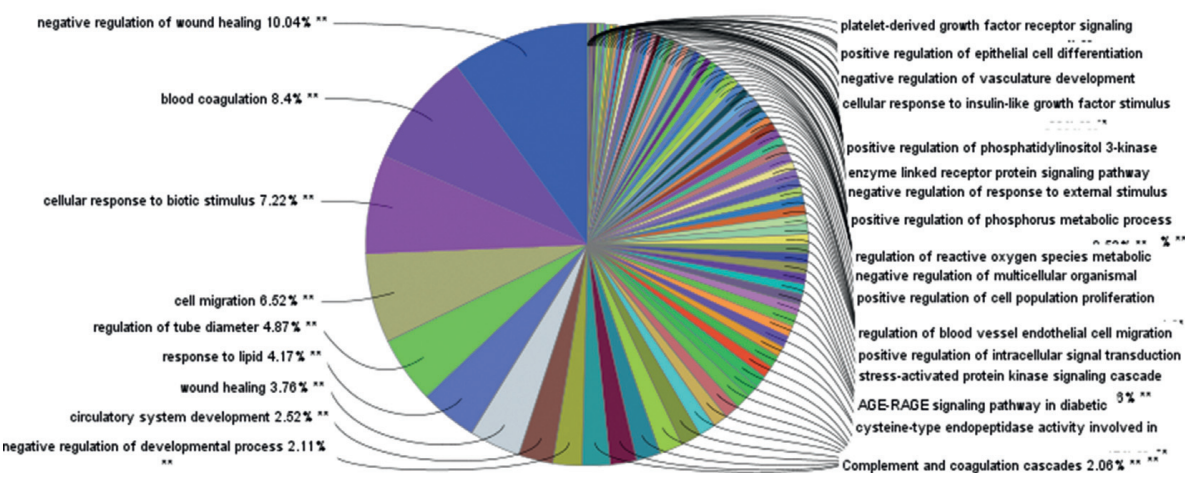

(f)

FIGURE 8: The results of enrichment analysis (a: biological processes; b: cell components; c: molecular function; d: signaling pathways; $X$-axis stands for fold enrichment. e and f: Cluego analysis). 
TABLE 4: DEPs with higher expression in brain tissue in the CI/CE operation comparison group.

\begin{tabular}{lcccccccccc}
\hline Gene name & Adrenal gland & Brain & Gastrocnemius & Heart & Kidney & Liver & Lung & Spleen & Testis & Thymus \\
\hline Ctsd & 297 & 167 & 154 & 126 & 258 & 125 & 651 & 195 & 35 & 182 \\
Sri & 28 & 45 & 11 & 16 & 68 & 13 & 100 & 146 & 144 & 85 \\
Lgals1 & 311 & 37 & 254 & 156 & 42 & 6 & 274 & 79 & 65 & 161 \\
Crp & 20 & 27 & 9 & 8 & 15 & 1447 & 27 & 19 & 21 & 24 \\
Rps6 & 30 & 24 & 18 & 18 & 22 & 24 & 50 & 145 & 29 & 98 \\
\hline
\end{tabular}

TABLE 5: DEPs with higher expression in brain tissue in the $\mathrm{CI} / \mathrm{CN}$ comparison group.

\begin{tabular}{lcccccccccc}
\hline Gene name & Adrenal gland & Brain & Gastrocnemius & Heart & Kidney & Liver & Lung & Spleen & Testis & Thymus \\
\hline Cst3 & 279 & 1558 & 273 & 279 & 240 & 112 & 722 & 495 & 381 & 638 \\
Tf & 42 & 447 & 41 & 24 & 43 & 18878 & 109 & 126 & 127 & 41 \\
Ppia & 183 & 308 & 37 & 54 & 217 & 181 & 207 & 269 & 236 & 413 \\
B2m & 685 & 186 & 166 & 558 & 507 & 1168 & 1661 & 1926 & 156 & 1003 \\
Car2 & 27 & 183 & 12 & 11 & 250 & 6 & 101 & 293 & 40 & 12 \\
Agt & 47 & 96 & 2 & 2 & 13 & 372 & 31 & 6 & 0.7 & 51 \\
Prdx6 & 108 & 68 & 28 & 59 & 103 & 79 & 292 & 41 & 46 \\
Gpx1 & 347 & 52 & 160 & 330 & 635 & 1326 & 375 & 669 & 27 \\
Aldh9a1 & 62 & 21 & 28 & 30 & 151 & 258 & 46 & 33 & 29 & 41 \\
Cat & 146 & 21 & 48 & 46 & 379 & 1270 & 113 & 81 & 8 & 56 \\
\hline
\end{tabular}

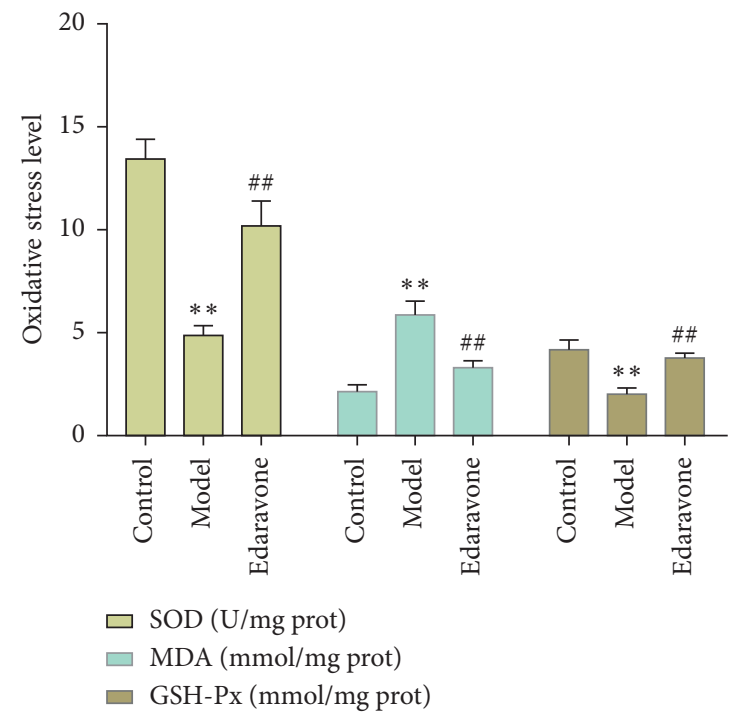

Figure 9: Oxidative stress indicators in brain tissue ( ${ }^{* *}$ compared with sham operation group, $P<0.05$; \#\#compared with model group, $P<0.05)$.

inflammation (GO:0050544 arachidonic acid binding), and other molecular functions. The cellular components focus on exosomes (GO:0070062 extracellular exosome), extracellular space (GO:0005615 extracellular space; GO: 0031012 extracellular matrix), blood particles (GO: 0072562 blood microparticle), intercellular adhesion (GO: 0005925 focal adhesion; 5913 cell-cell adherens junction), platelet (GO:0031093 platelet alpha granule lumen; GO: 0031089 platelet dense granule lumen), endoplasmic reticulum cavity (GO:0005788), and myelin sheath (GO:0043209).

Oxidative stress is considered to be an important factor leading to cell damage in acute stroke events. During the period of cerebral ischemia and perfusion, especially the reperfusion period, a large number of free radicals are produced, which attack the lipids and proteins of cell membranes $[34,35]$. In recent years, studies have found that in addition to directly attacking cell membranes and causing cell oxidative damage, free radicals can also participate in the process of neuronal damage by initiating apoptosis pathways. The free radicals produced after cerebral ischemia can act on mitochondria and change the permeability of mitochondrial pores. The depolarization of the mitochondrial membrane leads to the release of cytochrome $\mathrm{c}$ from mitochondria into the cytoplasm, which activates the caspase family to cause 
DNA damage and lead to apoptosis $[36,37]$. In addition, the reactive oxygen species produced by oxidative stress can also regulate the activity of nuclear transcription factor $\mathrm{NF}-\mathrm{kB}$ and affect the downstream genes of NF-kB, including iNOs, COX2, intercellular adhesion molecules, and cytokines [38-41]. They mediate the destruction of blood-brain barrier function and inflammation and play an important role in the signal transduction of apoptosis [42-44]. Therefore, after cerebral infarction and its reperfusion, the overproduction and burst of free radicals play a key role in the programmed death of neurons. Edaravone is an effective antioxidant, which can inhibit the oxidation of $\mathrm{NO}$ and increase the production of NO by increasing the expression of eNOS and inhibit the formation of peroxygen during reperfusion to improve or protect vascular blood flow [45]. According to recent reports, in MCAO model mice, edaravone administered within 6 hours of reperfusion can reduce infarct volume and improve neurological deficits. In the early stage of ischemic events, edaravone can inhibit the accumulation of 4-hydroxy2-nonenal (HNE) modified protein and 8-hydroxydeoxyguanosine $(8-\mathrm{OHdG})$ in the ischemic penumbra. In the later period, it reduces the activation of microglia, the expression of iNOS, and the formation of astrocytes peroxygen [46]. Jiao et al. found that edaravone can reduce the delayed neuronal death of the hippocampus after middle cerebral artery ischemia reperfusion, and its mechanism may be related to scavenging free radicals, resisting inflammation, and inhibiting the activation of astrocytes [47]. In terms of cerebral hemorrhage, edaravone may inhibit the activation of M1 type microglia and the accumulation of proinflammatory factors through the IRE1 $\alpha /$ TRAF2 signaling pathway after ER stress, reduce the edema and apoptosis of nerve cells after cerebral hemorrhage in mice, reduce the damage of white matter fiber bundles, and then protect nerve function [48]. In terms of the proliferation and differentiation of neural stem cells, edaravone can promote the proliferation of endogenous neural stem cells and astrocytes in the subventricular area of the ischemic injury side and the cerebral cortex around ischemia and promote the differentiation of endogenous neural stem cells into neurons [49]. Further studies have shown that edaravone promotes neuronal proliferation in acute stroke rats through the $\mathrm{Wnt} / \beta$-catenin signaling pathway and improves the neurological and cognitive functions of acute stroke rats [50]. In terms of iron metabolism, the effect of edaravone in repairing spinal cord injury in rats is achieved by regulating the $\mathrm{xCT} / \mathrm{GPX} 4 / \mathrm{ACSL} 4 / 5-\mathrm{LOX}$ iron death pathway and rebalancing the homeostasis of lipid oxidation. Further studies have shown that edaravone reduces 5-LOX metabolites to inhibit neuroinflammation, promote neuronal survival after spinal cord injury, and prevent iron death in neuronal cell lines [51]. Compared with previous studies, this study revealed the serum protein profile of edaravone in the treatment of cerebral infarction rats through serum TMT proteomics and discovered the relevant mechanism of edaravone regulating iron metabolism in cerebral infarction, which provides new ideas for the study of edaravone intervention in cerebral infarction and also provides reference information for future research on the mechanism of edaravone intervention in iron metabolism-related diseases.

\section{Conclusion}

The therapeutic mechanism of edaravone in the treatment of CI may involve platelet activation and aggregation, oxidative stress, intercellular adhesion, glycolysis and gluconeogenesis, iron metabolism, hypoxia, and so on. This study provides new reference for the clinical application of edaravone for CI.

\section{Data Availability}

The data that support the findings of this study are openly available in supplementary materials.

\section{Conflicts of Interest}

The authors declare no conflicts of interest.

\section{Authors' Contributions}

Guozuo Wang, Xiaomei Zeng, Shengqiang Gong, Shanshan Wang, Anqi Ge, Wenlong Liu, and Jinwen Ge are responsible for the study concept and design. Guozuo Wang, Xiaomei Zeng, Qi He, Shengqiang Gong, Shanshan Wang, Anqi Ge, Wenlong Liu, and Jinwen are responsible for the data collection, data analysis, and interpretation of network analysis; Guozuo Wang and Shengqiang Gong are responsible for the data collection, data analysis, and interpretation of experiments. Guozuo Wang drafted the paper; Jinwen Ge supervised the study; all the authors participated in the analysis and interpretation of data and approved the final paper. Guozuo Wang and Xiaomei Zeng should be considered joint first authors.

\section{Acknowledgments}

This work was supported by the National Natural Science Foundation of China (no. 81274008), the Key Project of Scientific Research Fund of Hunan Provincial Education Department (no. 19A378), the National Natural Science Foundation of Hunan Province, China (no. 2020JJ4467), and the National Key Research and Development Project of China (no. 2018YFC1704904).

\section{Supplementary Materials}

Table S1: edaravone potential targets and CI genes. Table S2: enrichment analysis results of proteomics. Table S3: enrichment analysis results of edaravone-CI PPI network (Supplementary Materials)

\section{References}

[1] H. Amani, E. Mostafavi, M. R. Alebouyeh et al., "Would colloidal gold nanocarriers present an effective diagnosis or treatment for ischemic stroke?" International Journal of Nanomedicine, vol. 14, pp. 8013-8031, 2019.

[2] R. A. Grysiewicz, K. Thomas, and D. K. Pandey, "Epidemiology of ischemic and hemorrhagic stroke: incidence, prevalence, mortality, and risk factors," Neurologic Clinics, vol. 26, no. 4, pp. 871-895, 2008. 
[3] W. Wang, B. Jiang, H. Sun et al., "Prevalence, incidence, and mortality of stroke in China," Circulation, vol. 135, no. 8, pp. 759-771, 2017.

[4] S. Hu, R. Gao, L. Liu et al., "Summary of "Chinese cardiovascular disease report 2018," Chinese Journal of Circulation, vol. 34, no. 3, pp. 209-220, 2019, in chinese.

[5] L. Wang, J. Wang, B. Peng, and Y. Xu, "Summary of "China stroke prevention and treatment report 2016"," Chinese Journal of Cerebrovascular Disease, vol. 14, no. 04, pp. 217224, 2017, in chinese.

[6] L. Sun, R. Clarke, R. Clarke et al., "Causal associations of blood lipids with risk of ischemic stroke and intracerebral hemorrhage in Chinese adults," Nature Medicine, vol. 25, no. 4, pp. 569-574, 2019.

[7] R. Rodrigo, R. Fernandez-Gajardo, R. Gutierrez et al., "Oxidative stress and pathophysiology of ischemic stroke: novel therapeutic opportunities," CNS \& Neurological DisorderDrug Targets, vol. 12, no. 5, pp. 698-714, 2013.

[8] Y.-C. Cheng, J.-M. Sheen, W. L. Hu, and Y.-C. Hung, "Polyphenols and oxidative stress in atherosclerosis-related ischemic heart disease and stroke," Oxidative Medicine and Cellular Longevity, vol. 2017, Article ID 8526438, 16 pages, 2017.

[9] S. Orellana-Urzúa, I. Rojas, L. Líbano, and R. Rodrigo, "Pathophysiology of ischemic stroke: role of oxidative stress," Current Pharmaceutical Design, vol. 26, no. 34, pp. 42464260, 2020.

[10] S. Lorenzano, N. S. Rost, M. Khan et al., "Early molecular oxidative stress biomarkers of ischemic penumbra in acute stroke,” Neurology, vol. 93, no. 13, pp. e1288-e1298, 2019.

[11] P. Narne, V. Pandey, and P. B. Phanithi, "Interplay between mitochondrial metabolism and oxidative stress in ischemic stroke: an epigenetic connection," Molecular and Cellular Neuroscience, vol. 82, pp. 176-194, 2017.

[12] S. Matsumoto, M. Murozono, M. Kanazawa, T. Nara, T. Ozawa, and Y. Watanabe, "Edaravone and cyclosporine A as neuroprotective agents for acute ischemic stroke," Acute Medicine \& Surgery, vol. 5, no. 3, pp. 213-221, 2018.

[13] S. Wu, E. Sena, K. Egan, M. Macleod, and G. Mead, "Edaravone improves functional and structural outcomes in animal models of focal cerebral ischemia: a systematic review," International Journal of Stroke, vol. 9, no. 1, pp. 101-106, 2014.

[14] J. Yang, X. Cui, J. Li, C. Zhang, J. Zhang, and M. Liu, "Edaravone for acute stroke: meta-analyses of data from randomized controlled trials," Developmental Neurorehabilitation, vol. 18, no. 5, pp. 330-335, 2015.

[15] Y. Yamamoto, "Plasma marker of tissue oxidative damage and edaravone as a scavenger drug against peroxyl radicals and peroxynitrite," Journal of Clinical Biochemistry \& Nutrition, vol. 60, no. 1, pp. 49-54, 2017.

[16] M. Frantzi, A. Latosinska, and H. Mischak, "Proteomics in drug development: the dawn of a new era?" ProteomicsClinical Applications, vol. 13, no. 2, Article ID 1800087, 2019.

[17] C. Monti, M. Zilocchi, I. Colugnat, and T. Alberio, "Proteomics turns functional," Journal of Proteomics, vol. 198, pp. 36-44, 2019.

[18] E. Z. Longa, P. R. Weinstein, and S. Carlson, "Reversible middle cerebral artery occlusion without craniectomy in rats," Stroke, vol. 20, no. 1, p. 84, 1989.

[19] X. Liu, S. Ouyang, B. Yu et al., "PharmMapper server: a web server for potential drug target identification using pharmacophore mapping approach," Nucleic Acids Research, vol. 38, pp. W609-W614, 2010.
[20] G. Stelzer, R. Rosen, I. Plaschkes et al., "The GeneCards suite: from gene data mining to disease genome sequence analysis," CurrProtoc Bioinformatics, vol. 54, 2016.

[21] A. Hamosh, A. F. Scott, J. S. Amberger, V. A. Bocchini, and C. A. McKusick, "Online Mendelian Inheritance in Man (OMIM), a knowledgebase of human genes and genetic disorders," Nucleic Acids Research, vol. 33, pp. D514-D517, 2005.

[22] D. Szklarczyk, A. Franceschini, S. Wyder, K. Forslund, D. Heller, and J. Huerta-Cepas, "STRING v10: protein-protein interaction networks, integrated over the tree of life," Nucleic Acids Research, vol. 43, pp. D447-D452, 2015.

[23] D. W. Huang, B. T. Sherman, and R. A. Lempicki, "Systematic and integrative analysis of large gene lists using DAVID bioinformatics resources," Nature Protocols, vol. 4, pp. 44-57, 2009.

[24] S. Miyake, H. Wakita, J. D. Bernstock et al., "Hypophosphorylation of ribosomal protein $\mathrm{S} 6$ is a molecular mechanism underlying ischemic tolerance induced by either hibernation or preconditioning," Journal of Neurochemistry, vol. 135, no. 5, pp. 943-957, 2015.

[25] W. S. Qu, Y. H. Wang, J. F. Ma et al., "Galectin-1 attenuates astrogliosis-associated injuries and improves recovery of rats following focal cerebral ischemia," Journal of Neurochemistry, vol. 116, no. 2, pp. 217-226, 2011.

[26] S. Ishibashi, T. Kuroiwa, M. Sakaguchi et al., "Galectin-1 regulates neurogenesis in the subventricular zone and promotes functional recovery after stroke," Experimental Neurology, vol. 207, no. 2, pp. 302-313, 2007.

[27] T. Nakase, T. Mizuno, S. Harada et al., "Angiotensinogen gene polymorphism as a risk factor for ischemic stroke," Journal of Clinical Neuroscience, vol. 14, no. 10, pp. 943-947, 2007.

[28] S. Wang, R. Zeng, L. Lei, and J. Huang, “Angiotensinogen gene polymorphism and ischemic stroke in East Asians: a meta-analysis," Neural Regen Research, vol. 8, no. 13, pp. 1228-1235, 2013.

[29] K. Gormley, S. Bevan, and H. S. Markus, "Polymorphisms in genes of the renin-angiotensin system and cerebral small vessel disease," Cerebrovascular Diseases, vol. 23, no. 2-3, pp. 148-155, 2007.

[30] M. Armogida, A. Spalloni, D. Amantea et al., "The protective role of catalase against cerebral ischemia in vitro and in vivo," International Journal of Immunopathology \& Pharmacology, vol. 24, no. 3, pp. 735-747, 2011.

[31] R. Nisticò, S. Piccirilli, M. L. Cucchiaroni et al., "Neuroprotective effect of hydrogen peroxide on an in vitro model of brain ischaemia," British Journal of Pharmacology, vol. 153, no. 5, pp. 1022-1029, 2008.

[32] G. E. Barreto, X. Sun, L. Xu, and R. G. Giffard, “Astrocyte proliferation following stroke in the mouse depends on distance from the infarct," PLoS One, vol. 6, no. 11, Article ID e27881, 2011.

[33] Y. F. Yang, Z. Chen, S. L. Hu et al., "Interleukin-1 receptor associated kinases-1/4 inhibition protects against acute hypoxia/ischemia-induced neuronal injury in vivo and in vitro," Neuroscience, vol. 196, pp. 25-34, 2011.

[34] P. H. Chan, "Role of oxidants in ischemic brain damage," Stroke, vol. 27, no. 6, pp. 1124-1129, 1996.

[35] L. Li, J. Tan, Y. Miao, P. Lei, and Q. Zhang, "ROS and autophagy: interactions and molecular regulatory mechanisms," Cellular and Molecular Neurobiology, vol. 35, no. 5, pp. 615-621, 2015.

[36] T. Sugawara, N. Noshita, A. Lewén et al., "Overexpression of copper/zinc superoxide dismutase in transgenic rats protects 
vulnerable neurons against ischemic damage by blocking the mitochondrial pathway of caspase activation," Journal of Neuroscience, vol. 22, no. 1, pp. 209-217, 2002.

[37] C. Michiels, E. Minet, D. Mottet, and M. Raes, "Regulation of gene expression by oxygen: NF-kappaB and HIF-1, two extremes," Free Radical Biology and Medicine, vol. 33, no. 9, pp. 1231-1242, 2002.

[38] C. T. D’Angio and J. N. Finkelstein, "Oxygen regulation of gene expression: a study in opposites," Molecular Genetics and Metabolism, vol. 71, no. 1-2, pp. 371-380, Article ID 11001829, 2000.

[39] Y. Ye, T. Jin, X. Zhang et al., "Meisoindigo protects against focal cerebral ischemia-reperfusion injury by inhibiting NLRP3 inflammasome activation and regulating microglia/ macrophage polarization via TLR4/NF- $\kappa \mathrm{B}$ signaling pathway," Frontiers in Cellular Neuroscience, vol. 13, p. 553, 2019.

[40] L. Ruan, F. Li, S. Li et al., "Effect of different exercise intensities on hepatocyte apoptosis in HFD-induced NAFLD in rats: the possible role of endoplasmic reticulum stress through the regulation of the IRE1/JNK and eIF $2 \alpha / \mathrm{CHOP}$ signal pathways," Oxidative Medicine and Cellular Longevity, vol. 2021, Article ID 6378568, 15 pages, 2021.

[41] B. Puig, S. Brenna, and T. Magnus, "Molecular communication of a dying neuron in stroke," International Journal of Molecular Sciences, vol. 19, no. 9, Article ID 2834, 2018.

[42] A. M. Pourbagher-Shahri, T. Farkhondeh, M. Talebi, D. M. Kopustinskiene, S. Samarghandian, and J. Bernatoniene, "An overview of NO signaling pathways in aging," Molecules, vol. 26, no. 15, p. 4533, 2021.

[43] M. Hedtjärn, A. L. Leverin, K. Eriksson, K. Blomgren, C. Mallard, and H. Hagberg, "Interleukin-18 involvement in hypoxic-ischemic brain injury," Journal of Neuroscience, vol. 22, no. 14, pp. 5910-5919, 2002.

[44] G. del Zoppo, I. Ginis, J. M. Hallenbeck, C. Iadecola, X. Wang, and G. Z. Feuerstein, "Inflammation and stroke: putative role for cytokines, adhesion molecules and iNOS in brain response to ischemia," Brain Pathology, vol. 10, no. 1, pp. 95-112, 2000.

[45] H. Yoshida, H. Yanai, Y. Namiki, K. Fukatsu-Sasaki, N. Furutani, and N. Tada, "Neuroprotective effects of edaravone: a novel free radical scavenger in cerebrovascular injury," CNS Drug Reviews, vol. 12, no. 1, pp. 9-20, 2006.

[46] N. Zhang, M. Kominc-Kobayashi, R. Tanaka, M. Liu, Y. Mizuno, and T. Urabc, "Edaravone reduces early accumulation of oxidative products and sequentialinflammatory responses after transient focal ischemia in mice brain," Stroke, vol. 36, pp. 2220-2225, 2005.

[47] L. Jiao, The Role of Edaravone in Hippocampal Delayed Neuronal Death and Distant Memory Function after Middle Cerebral Artery Ischemia-Reperfusion in Rats and its mechanism, Huazhong University of Science and Technology, Wuhan, China, 2011.

[48] S. H. Jiang, Effects of Edaravone on Inflammatory Response and Endoplasmic Reticulum Stress Pathway after Cerebral hemorrhage, Zunyi Medical University, Zunyi, China, 2020.

[49] X. Y. Liang, Y. Gu, M. Zhao et al., "Edaravone intervenes in the proliferation and differentiation of neural stem cells in rats with permanent cerebral ischemia," Journal of Anatomy, vol. 52, no. 3, pp. 370-376, 2021.

[50] F. Wang, Y. Wang, and B. Zhao, "Effects of edaravone based on $\mathrm{Wnt} / \beta$-catenin signaling pathway on neuronal proliferation in rats with acute stroke," Journal of Brain and Neurological Disorders, vol. 29, no. 7, pp. 406-411, 2021.

[51] X. Wang, Experimental Study on the Regulation of Iron Death by Edaravone to Repair Spinal Cord injury, Tianjin Medical University, Tianjin, China, 2020. 